\title{
THE CHARACTERS OF THE FINITE GENERAL LINEAR GROUPS
}

\author{
BY \\ J. A. GREEN
}

Introduction. In this paper we show how to calculate the irreducible characters of the group $G L(n, q)$ of all nonsingular matrices of degree $n$ with coefficients in the finite field of $q$ elements. These characters have been given for $n=2$ by H. Jordan [8], Schur [10], and others, and for $n=3$ and $n=4$ by Steinberg [12], who has also [13] done important work in the general case.

We are concerned here with "ordinary" characters, that is, characters of representations by matrices with complex coefficients. Let $\chi_{1}, \cdots, \chi_{h}$ be the distinct absolutely irreducible ordinary characters of a group \&f of order $g$. By a character of \&S (often called a "generalised character" or "difference character") we mean a class-function $\phi$ on (S) of the form

$$
\phi=\sum_{i=1}^{h} a_{i} \chi_{i}
$$

where the $a_{i}$ are rational integers. If the $a_{i}$ are non-negative integers, so that $\phi$ is the character of a matrix representation of $\$, \phi$ will be called a proper character.

For any two complex-valued class-functions $\chi, \psi$ on $\$$, define the "scalar product"

$$
(\chi, \psi)=\frac{1}{g} \sum_{x \in \Theta} \chi(x) \Psi(x),
$$

and "norm"

$$
\|x\|=(x, x) \text {. }
$$

We have two classical principles for calculating the irreducible characters of (B):

$A$ character $\chi$ of $\&$ is irreducible if and only if $\|\chi\|=1$, and $\chi(1)>0$ (we use 1 for the identity element of $(\xi)$, and

Two irreducible characters $\chi, \psi$ of $\$$ are distinct if and only if $(\chi, \psi)=0$.

Let $q$ be a fixed prime-power, and write $\mathfrak{S}_{n}=G L(n, q)$. Characters of $\mathbb{S}_{n}$ are derived from two sources; from the subgroups of $\oiint_{n}$ on the one hand, and from the defining representation of $\mathfrak{S}_{n}$ (this is of course a modular representation) on the other. The use of subgroups is based on Frobenius' treatment (Frobenius [5]) of the characters of the symmetric group $\widetilde{S}_{n}$ (cf. Steinberg [13]). Let $V_{n}$ be the space of $n$-vectors with components in $\mathfrak{F}=G F(q)$, the

Received by the editors September 7, 1954. 
finite field of $q$ elements. An element $A \in \mathcal{G}_{n}$ is a linear operator on $V_{n}$ by the usual product $v A\left(v \in V_{n}\right)$.

Let $n=s_{1}+s_{2}+\cdots+s_{k}$ be a partition of $n$ into positive integers $s_{i}$, and let $V^{(i)}$ be the subspace of $V_{n}=V^{(0)}$ consisting of vectors whose first $s_{1}+s_{2}$ $+\cdots+s_{i}$ components are zero. Then the subgroup $\mathfrak{S}_{s_{1} s_{2}} \cdots_{s_{k}}$ of all elements of $\mathcal{B S}_{n}$ which leave invariant the chain of subspaces

$$
V^{(0)}>V^{(1)}>\cdots>V^{(k)}=0
$$

consists of all matrices

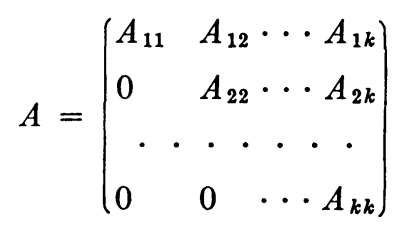

for which $A_{i i} \in \oiint_{s_{i}}(i=1,2, \cdots, k)$.

If $\alpha_{i}$ is a character of $\mathcal{B}_{s_{i}}(i=1,2, \cdots, k)$, then it is clear that $\psi(A)$ $=\alpha_{1}\left(A_{11}\right) \cdots \alpha_{k}\left(A_{k k}\right)$ is a character of $\mathfrak{W}_{s_{1} s_{2}} \cdots s_{k}$. We define $\alpha_{1} \circ \alpha_{2} \circ \cdots \circ \alpha_{k}$ to be the character of $\mathbb{S}_{n}$ which is induced by $\psi$. It is shown in $\$ 2$ that the binary multiplication $\alpha_{1} \circ \alpha_{2}$ is commutative, associative, and bilinear, and that $\alpha_{1} \circ \alpha_{2} \circ \cdots \circ \alpha_{k}$ is equal to the $k$-fold product $\left(\cdots\left(\alpha_{1} \circ \alpha_{2}\right) \circ \cdots \circ \alpha_{k}\right)$. Let $\mathcal{A}_{n}$ be the space of all class-functions on $\mathfrak{S}_{n}(n=1,2, \cdots)$, and let $\mathcal{A}$ be the (restricted, or weak) direct sum $\sum_{n=1}^{\infty} \mathcal{A}_{n}$. Since Frobenius' induced character formula can be applied to any class-function $\psi$, we may define $\alpha_{1} \circ \alpha_{2}$, as above, for any $\alpha_{1} \in \mathcal{A}_{s_{1}}, \alpha_{2} \in \mathcal{A}_{s_{2}}$, and extending this definition by linearity, $A$ becomes a commutative, associative algebra over the complex field 5 .

This o-product generates characters of $\mathbb{B}_{n}$ from those of the groups $\mathfrak{S}_{m}(m<n)$. To start this inductive process and to keep it going, we need our second construction. This is the following theorem, which is based on R. Brauer's fundamental characterisation of characters of finite groups (Brauer [1]).

Write $M(\mathfrak{F})$ for the multiplicative group of any field $\mathfrak{F}$, and $\mathfrak{C}$ for the field of complex numbers.

THEOREM 1. Let $x \rightarrow R(x)(x \in(S)$ be any representation of a finite group (B) by nonsingular $n \times n$ matrices $R(x)$ with coefficients in a finite field $\mathfrak{F}^{*}$. Suppose that $\mathfrak{F}^{*}$ contains, for each $x \in \mathfrak{B}$, the latent roots

$$
\xi_{1}(x), \cdots, \xi_{n}(x)
$$

of $R(x)$. Then if

$$
\theta: \alpha \rightarrow \alpha_{0} \quad\left(\alpha \in M\left(\mathfrak{F}^{*}\right)\right)
$$

is any homomorphism of $M\left(\mathfrak{F}^{*}\right)$ into $M(\mathfrak{S})$, and if $S\left(t_{1}, \cdots, t_{n}\right)$ is any sym- 
metric polynomial in $t_{1}, \cdots, t_{n}$ with rational integer coefficients, the function

$$
\chi(x)=S\left(\xi_{1}(x)_{0}, \cdots, \xi_{n}(x)_{0}\right)
$$

is a character of (5).

This theorem is proved in $\S 3$.

The nature of the characters of $\mathbb{S}_{n}$, as functions on $\mathbb{S}_{n}$, is of great interest. All characters are what I have called uniform functions (Definition 4.12). This means that the value at any conjugacy class of $\mathbb{S}_{n}$ is given, by a certain fixed "degeneracy rule," in terms of the functions which describe the character on the classes of principal matrices; these are the matrices whose latent roots are all distinct. For example, the different conjugacy classes of $\mathbf{S}_{2}$ are typified by matrices with the following canonical forms:

Class

$$
\text { Typical matrix } \quad\left(\begin{array}{ll}
\alpha & \\
& \alpha
\end{array}\right) \quad\left(\begin{array}{ll}
\alpha & 1 \\
& \alpha
\end{array}\right)
$$

$$
c_{3}
$$$$
\left(\begin{array}{ll}
\alpha & \\
& \beta
\end{array}\right) \quad\left(\begin{array}{ll}
\eta & \\
& \eta^{q}
\end{array}\right),
$$

where $\alpha, \beta \in M(G F(q))$ and $\alpha \neq \beta$, while $\eta, \eta^{\natural} \in M\left(G F\left(q^{2}\right)\right)$ are roots of an irreducible polynomial over $G F(q)$ of degree 2. A uniform function $U$ on $\mathfrak{G}_{2}$ is characterized by two independent functions ("principal parts"): $U^{\prime}(\alpha, \beta)$, which is defined for $\alpha, \beta \in M(G F(q))$ and is symmetric in $\alpha, \beta$, and $U^{\prime \prime}(\eta)\left({ }^{1}\right)$, defined for $\eta \in M\left(G F\left(q^{2}\right)\right)$ and satisfying. $U^{\prime \prime}(\eta)=U^{\prime \prime}\left(\eta^{q}\right)$. The values on the different classes are then given by

$$
\begin{aligned}
& U\left(c_{1}\right)=(q+1) U^{\prime}(\alpha, \alpha) / 2+(-q+1) U^{\prime \prime}(\alpha) / 2, \\
& U\left(c_{2}\right)=U^{\prime}(\alpha, \alpha) / 2+U^{\prime \prime}(\alpha) / 2, \\
& U\left(c_{8}\right)=U^{\prime}(\alpha, \beta), \\
& U\left(c_{4}\right)=U^{\prime \prime}(\eta) .
\end{aligned}
$$

In the degeneracy rule occur polynomials $Q_{\rho}^{\lambda}(q)$, defined for each pair $\rho, \lambda$ of partitions of each positive integer $n$. These are expressed in terms of further polynomials which were first introduced by $P$. Hall (in unpublished lectures, Hall [7]); the $Q_{p}^{\lambda}(q)$ are closely connected with the characters $\chi_{\rho}^{\lambda}$ of the symmetric group $\mathfrak{S}_{n}$.

The uniform functions on $\mathcal{S}_{n}$, for $n=1,2, \cdots$, generate a subspace $V$ of the algebra $\mathcal{A}$. Theorems 7 and $8(\S 5)$ show respectively that $V$ is a subalgebra of $\mathcal{A}$, and that $U$ contains the characters which are provided by Theorem 1. Theorem $11(\$ 6)$ gives, with the aid of certain remarkable orthogonality relations (Theorem 10) satisfied by the $Q_{\rho}^{\lambda}(q)$, a formula for the scalar product of two uniform functions. The simplicity of this formula is the chief technical advantage from introducing this type of function. 4.12).

(1) We use a slightly different notation for $U^{\prime}$ and $U^{\prime \prime}$ in the general case (see Definition 
In Theorem $14(\$ 8)$ we give explicit formulae for all the irreducible characters of $\oiint_{n}$, in terms of certain "basic characters," whose values at any class can in turn be computed by the degeneracy rule. A complete table of characters can therefore be written down as soon as the polynomials $Q_{p}^{\lambda}(q)$ are known. Tables of $Q_{\rho}^{\lambda}(q)$ for partitions $\rho, \lambda$ of $n=1,2,3,4,5$ are given in an Appendix. Theorem 14 includes also explicit formulae for the degrees of all the irreducible characters.

There is an extremely complete duality between the rows and columns of the character table of $\mathbb{S}_{n}$. The rule given in Theorem 14 , by which the characters are obtained from the basic characters, is in effect a dual degeneracy rule which involves the characters $\chi_{\rho}^{\lambda}$ of the symmetric group $\widetilde{S}_{n}$ in place of the $Q_{\rho}^{\lambda}(q)$.

I should like to express my gratitude to Professor P. Hall for permission to use two unpublished theorems of his (Theorems 3 and 4) and also to Dr. W. Ledermann, who read the manuscript and made many valuable suggestions.

1. Notation, conjugacy classes, and class types. Let $\mathfrak{F}=\mathfrak{F}_{1}$ be the Galois field $G F(q)$ with $q$ elements. Write $\mathfrak{F}_{d}=G F\left(q^{d}\right)(d=1,2, \cdots)$. We regard each $\mathfrak{F}_{d}$ as an extension of $\mathfrak{F}=\mathfrak{F}_{1}$, and we can think of the $\mathfrak{F}_{d}$, for $1 \leqq d \leqq n$, as subfields of $\mathfrak{F}^{*}=\mathfrak{F}_{n !}$. The automorphisms of $\mathfrak{F}^{*}$ over $\mathfrak{F}$ are the mappings

$$
\alpha \rightarrow \alpha^{q^{r}} \quad\left(\alpha \in \mathfrak{F}^{*}\right)
$$

for $r=0,1, \cdots, n !-1$, the elements $\alpha$ of degree $d$ (over $\mathfrak{F}$ ) are those for which exactly $d$ of the conjugates

$$
\alpha, \alpha^{q}, \alpha^{q^{2}}, \cdots
$$

are distinct, and the subfield $\mathfrak{F}_{d}$ consists of 0 together with all elements of degree dividing $d$. (The zero element 0 is conventionally excluded from the set of elements of degree 1.) The roots of an irreducible polynomial $f(t)$ over $\mathfrak{F}$ of degree $d \leqq n$ are regarded as being in our universal field $\mathfrak{F}^{*}$; they form of course a set of conjugate elements

$$
\alpha, \alpha^{q}, \cdots, \alpha^{q^{d-1}}
$$

of degree $d$ (unless $f(t)=t$ ).

Two matrices $A, B \in \mathbb{S}_{n}$ are conjugate in $\mathfrak{S}_{n}$ if and only if they are similar over $\mathfrak{F}$ (for which we shall write $A \sim B$ ) in the usual sense of matrix theory. Let $f(t)=t^{d}-a_{d-1} t^{d-1}-\cdots-a_{0}$ be a polynomial over $\mathfrak{F}$, of degree $d$. Define the matrices

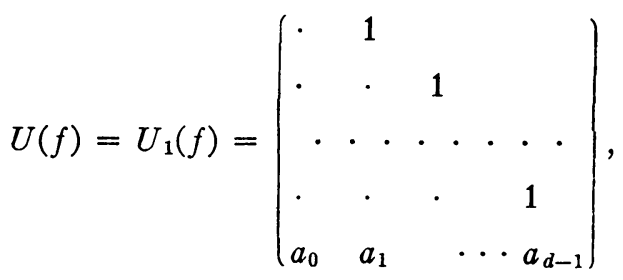




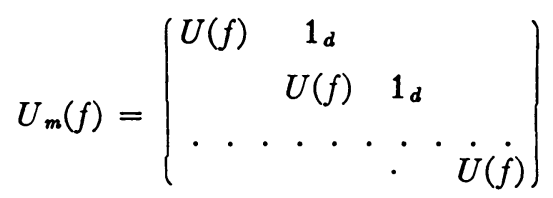

with $m$ diagonal blocks $U(f)$, and $1_{d}$ is the identity matrix of $G_{d}$, and finally, if $\lambda=\left\{l_{1}, l_{2}, \cdots, l_{p}\right\}$ is a partition of a positive integer $k$ whose $p$ parts, written in descending order, are $1_{1} \geqq 1_{2} \geqq \cdots \geqq 1_{p}>0$,

$$
U_{\lambda}(f)=\operatorname{diag}\left\{U_{l_{1}}(f), U_{l_{2}}(f), \cdots, U_{l_{p}}(f)\right\} .
$$

The characteristic polynomial $\left|t 1-U_{\lambda}(f)\right|$ is $f(t)^{k}$. Let $A \in \mho_{n}$ have characteristic polynomial

$$
f_{1}^{k_{1}} f_{2}^{k_{2}} \cdots f_{N}^{k_{N}}
$$

where $f_{1}, f_{2}, \cdots, f_{N}$ are distinct irreducible polynomials over $\mathfrak{F}, k_{i} \geqq 0$ $(i=1,2, \cdots, N)$ and, if $d_{1}, d_{2}, \cdots, d_{N}$ are the respective degrees of $f_{1}, f_{2}, \cdots, f_{N}, \sum_{i=1}^{N} k_{i} d_{i}=n$. Then we have $\left({ }^{2}\right)$

$$
A \sim \operatorname{diag}\left\{U_{\boldsymbol{\nu}_{1}}\left(f_{1}\right), U_{\boldsymbol{\nu}_{\mathbf{2}}}\left(f_{2}\right), \cdots, U_{\boldsymbol{\nu}_{N}}\left(f_{N}\right)\right\}
$$

where $\nu_{1}, \nu_{2}, \cdots, \nu_{N}$ are certain partitions of $k_{1}, k_{2}, \cdots, k_{N}$ respectively. By convention 0 is the only partition of the integer 0 , and in the formula above, a term $U_{0}(f)$ is to be omitted. We may denote the conjugacy class $c$ of $A$ by the symbol

$$
c=\left(f_{1}^{\nu_{1}} f_{2}^{\nu_{2}} \cdots f_{N}^{N_{N}}\right) .
$$

For many purposes, however, we shall prefer the following more systematic description of classes. Let $F$ be the set of irreducible polynomials $f=f(t)$ over $\mathfrak{F}$, of degrees $\leqq n$, excepting the polynomial $t$. Write $d(f)$ for the degree of $f \in F$. The class $c$ is described by the function $\nu_{c}(f)$ on $F$, whose value $\nu_{c}(f)$ is the partition (possibly zero) with which $f$ is associated in the canonical form of a matrix of $c$ (as, for example, $f_{i}$ is associated with $\nu_{i}$ in the case above).

Let us write $|\nu|$ for the integer of which $\nu$ is a partition. Then we see at once

LemMa 1.1. Let $\nu(f)$ be a partition-valued function on ${ }^{\circ}$. Then there exists a class $c$ of $\oiint_{n}$ such that $\nu(f)=\nu_{c}(f)($ each $f \in F)$ if and only if

(2) Cf. Jacobson, Lectures in abstract algebra, vol. II. The canonical form displayed there differs from ours, in that the matrix which corresponds to our $U_{m}(f)$ (see Jacobson, p. 97) has, in place of $1_{d}$, a $d \times d$ matrix whose coefficients are all zero, except for a coefficient unity in one corner. However our matrix is similar to Jacobson's, provided that the roots of the irreducible polynomial $f(t)$ are all distinct, which is certainly the case when the coefficients are in a finite field. 


$$
\sum_{f \in F}|\nu(f)| d(f)=n .
$$

We shall denote the class $c$ by the symbol

$$
c=\left(\cdots f^{v(f)} \cdots\right) \text {. }
$$

By the latent roots, characteristic polynomial of a class $c$, we mean the corresponding things for a matrix of $c$. A class $c$ is principal if each $\nu_{c}(f)=\{1\}$ or 0 ; alternatively, the characteristic polynomial $F(t)$ of $c$ has no repeated factors, so that the latent roots of $c$ are all distinct. If $F(t)$ has $r_{d}$ factors of degree $d(d=1,2, \cdots, n)$ we say $c$ is of principal type $\rho, \rho$ being the partition $\left\{1^{r_{1}} 2^{r_{2}} \cdots n^{r_{n}}\right\}$ of $n=r_{1}+2 r_{2}+\cdots+n r_{n}$ which has $r_{1}$ parts $1, r_{2}$ parts $2, \cdots$. A class $c$ is primary if $\nu_{c}(f)=0$ except for one particular $f \in F ; c$ has the form $\left(f^{\nu}\right)$ for some partition $\nu$. Primary classes $\left(f^{\lambda}\right)$ and $\left(g^{\mu}\right)(f, g \in F)$ are said to have the same type if $\lambda=\mu$ and $d(f)=d(g)$.

In general, let $c=\left(\cdots f^{v(c)} \cdots\right)$. Let $d$ be a positive integer, and let $\nu$ be a partition other than zero. Let $r_{c}(d, \nu)$ be the number of $f \in F$ of degree $d$, for which $\nu_{c}(f)=\nu$. Let $\rho_{c}(\nu)$ be the partition

$$
\left\{1^{r_{c}(1, v)} 2^{r_{c}(2, v)} \cdots\right\} \text {. }
$$

Then two classes $b, c$ are of the same type if and only if $\rho_{b}(\nu)=\rho_{c}(\nu)$ for each nonzero partition $\nu$. For example, all the matrices

$$
\left(\begin{array}{lll}
\alpha & & \\
& \beta & 1 \\
& & \beta
\end{array}\right) \quad\left(\alpha, \beta \in M\left(\mathfrak{F}_{1}\right)\right),
$$

are of the same type in $\mathfrak{S}_{3}$, although of course each new pair $(\alpha, \beta)$ describes a new class. Here $\rho(\{1\})=\rho(\{2\})=\{1\}$, and $\rho(\nu)=0$ for $\nu \neq\{1\},\{2\}$.

Let $\rho(\nu)$ be a partition-valued function on the nonzero partitions $\nu(\rho(\nu)$ may take the value 0$)$. The condition for $\rho(\nu)$ to describe a type of $\Theta_{n}$ is

$$
\sum_{\nu}|\rho(\nu)||\nu|=n \text {. }
$$

For example, $\rho(\nu)$ describes the principal type $\rho$ if $\rho(\nu)=0(\nu \neq\{1\})$, and $\rho(\{1\})=\rho$. The number $t(n)$ of functions $\rho(\nu)$ satisfying (1) is naturally independent of $q$, and is the number of types of classes of $\mathbb{B}_{n}$. For sufficiently large $q$ there will be classes of every type, but for small values of $q$ certain types may not be represented. For example, if $q=2$, there is no class of principal type $\left\{1^{3}\right\}$, because there are no matrices

$$
\left(\begin{array}{lll}
\alpha & & \\
& \beta & \\
& & \gamma
\end{array}\right)
$$

in which $\alpha, \beta, \gamma$ are in $\mathfrak{F}$ and are distinct. 
The number $t(n)$ appears as the number of rows or columns of a character table of $\mathrm{BS}_{n}$. This is because the irreducible characters, which by a wellknown theorem of representation theory are the same in number as the conjugacy classes, themselves collect into types in a corresponding way, and the values of all the characters of a given type at all the classes of a given type can be included in a single functional expression.

It is not hard to show that $t(n)$ is the coefficient of $x^{n}$ in the power-series expansion of

$$
p(x)^{p_{1}} p\left(x^{2}\right)^{p_{2}} p\left(x^{3}\right)^{p_{3}} \ldots
$$

where $p_{n}$ is the number of partitions of $n$, and $p(x)$ is the partition function $\sum_{n=0}^{\infty} p_{n} x^{n}=1 /(1-x)\left(1-x^{2}\right) \cdots$. We suppress the proof, because the result is not required for our investigation of the characters of $\mathfrak{S}_{n}$, but give below some values of $t(n)$; these are most easily calculated with the help of this formula.

$$
\begin{array}{rllllcc}
n=1 & 2 & 3 & 4 & 5 & 6 & 7 \\
t(n)=1 & 4 & 8 & 22 & 42 & 103 & 199 .
\end{array}
$$

For the number $c(n, \dot{q})$ of classes of $\oiint_{n}=G L(n, q)$, there is a similar generating function

$$
\sum_{n=0}^{\infty} c(n, q) x^{n}=\prod_{d=1}^{\infty} p\left(x^{d}\right)^{w(d, q)}
$$

where

$$
w(d, q)=\frac{1}{d} \sum_{k \mid d} \mu(k) q^{d / k}
$$

is the number of irreducible polynomials $f(t)$ of degree $d$ over $G F(q)$. We see from this that for given $n, c(n, q)$ is a polynomial in $q$ with constant rational coefficients.

2. Hall's polynomials. Induced characters. A module $(V, \Omega)$ is an abelian group $V$ equipped with a set $\Omega$ of endomorphic operators. We shall assume also that $\Omega$ contains the identity automorphism of $V$. Two modules $(V, \Omega)$, $\left(V^{\prime}, \Omega^{\prime}\right)$ are equivalent if the abelian groups $V, V^{\prime}$ are isomorphic (we shall then identify $V$ and $V^{\prime}$ ), and $\Omega$ and $\Omega^{\prime}$ generate the same ring of endomorphisms of $V$. It is clear that equivalent modules have isomorphic submodule lattices, and isomorphic automorphism groups.

Let $A$ be any $n \times n$ matrix with coefficients in $\mathfrak{F}$. Write $V_{A}=V_{A}(\mathfrak{F})$ for the module which has $V=V_{n}$ as abelian group, and for operators the scalar multiplications $\mathfrak{F}$, together with $A$. Write $V_{\lambda}=V_{\lambda}(q)=V_{U_{\lambda}(f)}(\mathfrak{F})$, where $f(t)=t$, and $\lambda$ is any nonzero partition; we may also define $V_{0}(q)=0$. It is 
clear that $V_{\lambda}$ is equivalent to $V_{U_{\lambda}(l)}$, where $l=t-\alpha(\alpha \in \mathfrak{F})$ is any linear polynomial over $\mathfrak{F}$. Next let $f \in F$ have degree $d>1$. The matrix $U(f)$ generates, in the algebra of linear transformations of $V_{d}$, a field $\mathfrak{F}_{d}$. We then see at once that $V_{U_{\lambda}(f)}(\mathfrak{F})$ is equivalent to $V_{\lambda}\left(q^{d}\right)$.

Suppose now that $A \in \mathbb{S}_{n}$ and that $A$ is in the class $c=\left(f_{1}^{\nu_{1}} f_{2}^{\nu_{2}} \cdots f_{N}^{\nu_{N}}\right)$. Matrices which are similar determine isomorphic modules, so we may write $V_{A}=V_{c}$ in what follows, without ambiguity. Since $A \sim \operatorname{diag}\left(A_{1}, A_{2}, \cdots\right.$, $\left.A_{N}\right)$ where $A_{i}=U_{v_{i}}\left(f_{i}\right)(i=1,2, \cdots, N), V_{c}$ is isomorphic to the direct sum

$$
V_{A_{1}} \oplus V_{A_{2}} \oplus \cdots \oplus V_{A_{N}}
$$

and since $f_{1}, f_{2}, \cdots, f_{N}$ are distinct, this is the unique decomposition of $V_{c}$ into indecomposable characteristic submodules.

Write $L(c), L_{\lambda}(q)$ respectively for the lattices of submodules of $V_{c}, V_{\lambda}(q)$, and let $A(c), A_{\lambda}(q)$ be the groups of automorphisms of the same modules. We have now

Lemma 2.1. If $c=\left(\cdots f^{v(f)} \cdots\right)$, then

$$
L(c) \cong \prod_{f \in F} L_{\nu(f)}\left(q^{d(f)}\right),
$$

and

$$
A(c) \cong \prod_{f \in F} A_{\nu(f)}\left(q^{d(f)}\right) .
$$

(I in each case stands for direct product, in the usual sense of abstract algebra.)

This lemma shows that $L(c), A(c)$ depend only on the type of the conjugacy class $c$.

The group $A(c)$ is just the centralizer in $B_{n}$ of an element $A \in c$. Let $a(c)$ be its order, and let $a_{\lambda}(q)$ be the order of $A_{\lambda}(q) ; a_{\lambda}(q)$ is therefore the order of the centralizer of the matrix $U_{\lambda}=U_{\lambda}(f)$, where $f(t)=t$. It can be shown that

$$
a_{\lambda}(q)=q^{|\lambda|+2 n_{\lambda}} \sum_{i=1}^{s} \phi_{k i-k i+1}\left(\frac{1}{q}\right)\left({ }^{3}\right),
$$

where $k_{1} \geqq k_{2} \geqq \cdots \geqq k_{s}>0$ are the parts of the partition conjugate to $\lambda$, and $k_{s+1}=0$ (see, for example, Littlewood [9]), and by

Definition 2.2. $\phi_{r}(t)=(1-t)\left(1-t^{2}\right) \cdots\left(1-t^{r}\right)$ if $r \geqq 1, \phi_{0}(t)=1$;

$\left(^{3}\right)$ Hall [7]. This formula is easily obtained by considering the centralizer algebra $C$ of the of the matrix $U_{\lambda}$, whose dimension Frobenius has given by an expression (see, for example, Wedderburn, Lectures on matrices, or Jacobson, Lectures in abstract algebra, vol. II) which may be reduced to $|\lambda|+2 n_{\lambda}$. It is not hard to see that the quatient of $C$ by its radical is the sum of total matrix algebras of degrees $k_{1}-k_{2}, k_{2}-k_{3}, \cdots, k_{s}$, respectively, and from this the order $a_{\lambda}(q)$ of the group $A_{\lambda}(q)$ of units of $C$ may be written down. 
Definition 2.3. If the conjugate partition of $\lambda$ has parts $k_{1}, k_{2}, \cdots, k_{s}$, then $n_{\lambda}=\sum_{i=1}^{s} C_{k_{i}, 2}$.

We have then

Lemma 2.4. If $c=\left(\cdots f^{\nu(f)} \cdots\right)$, then $a(c)=\prod_{f \in F} a_{p(f)}\left(q^{d(f)}\right)$, where $a_{\lambda}(q)$ is the polynomial defined above, of degree $|\lambda|+2 n_{\lambda}$ in $q$.

We consider next the character $\chi=\alpha_{1} \circ \alpha_{2} \circ \cdots \circ \alpha_{k}$ described in the introduction. The character $\psi^{*}$ of a group $\$ S$ induced by the character $\psi$ of a subgroup $\mathfrak{S}$ which has cosets

$$
\mathfrak{S} G_{1}=\mathfrak{S}, \mathfrak{S} G_{2}, \cdots \quad\left(G_{1}, G_{2}, \cdots \in(\mathfrak{S})\right.
$$

is given by the formula (Frobenius [3])

$$
\psi^{*}(A)=\sum_{i} \psi\left(G_{i} A G_{i}^{-1}\right)
$$

summed over those cosets $\mathfrak{S} G_{i}$ for which $G_{i} A G_{i}^{-1} \in \mathfrak{E}$, that is, for which $\mathfrak{S C} G_{i} A=\mathfrak{S} G_{i}$.

In our case the cosets (2) are in 1-1 correspondence with the different chains

$$
V^{(0)}>V^{(1)}>\cdots>V^{(k-1)}>V^{(k)}=0
$$

of subspaces of $V=V^{(0)}$, which have factors of dimensions $s_{1}, s_{2}, \cdots, s_{k}$ respectively. If $\mathfrak{S} G A=\mathfrak{S} G$, all the subspaces in the chain (3) which corresponds to $\mathfrak{S} G$ are closed to $A$, which means that they are submodules of $V_{A}$. The matrix $G A G^{-1}$ lies in $\mathfrak{S}=\mathfrak{S}_{s_{1} \varepsilon_{2}} \cdots_{s_{k}}$, and if

$$
G A G^{-1}=\left(\begin{array}{cccc}
A_{11} & A_{12} & \cdots & A_{1 k} \\
& A_{22} & \cdots & A_{2 k} \\
\cdots & \cdots & \cdot \\
& & A_{k k}
\end{array}\right) \quad\left(A_{i i} \in \mathcal{G}_{s_{i}}\right),
$$

then the factor module $V^{(i-1)} / V^{(i)}$ of $V_{A}$ is isomorphic to $V_{A_{i i}}(i=1,2, \cdots$, $k)$.

Thus we get the following

THEOREM 2. Let $\alpha_{1}, \cdots, \alpha_{k}$ be characters of $\mathcal{S}_{s_{1}}, \cdots, \mathcal{S}_{s_{k}}$ respectively, where $s_{1}, \cdots, s_{k}$ are positive integers such that $s_{1}+\cdots+s_{k}=n$. Then the value of the character $\alpha_{1} \circ \cdots \circ \alpha_{k}$ of $\mathfrak{G}_{n}$ at a class $c$ is

$$
\left(\alpha_{1} \circ \cdots \circ \alpha_{k}\right)(c)=\sum g_{c_{1}}^{c} \cdots c_{k} \alpha_{1}\left(c_{1}\right) \cdots \alpha_{k}\left(c_{k}\right),
$$

where the summation is over all rows $c_{1}, \cdots, c_{k}$ of classes respectively of $\mathcal{B}_{s_{1}}, \cdots, B_{s_{k}}$, and $g_{c_{1} \cdots c_{k}}^{c}$ is the number of chains (3) of submodules of a module $V^{(0)}=V_{c}$ in which $V^{(i-1)} / V^{(i)}$ is isomorphic to $V_{c_{i}}(i=1,2, \cdots, k)$. 
We remarked in the introduction that $\alpha_{1} \circ \cdots \circ \alpha_{k}$ can be defined if $\alpha_{1}, \cdots, \alpha_{k}$ are any complex-valued class-functions on $\mathcal{B}_{s_{1}}, \cdots, B_{s_{k}}$ respectively; it is clear that formula (4) still applies. We prove in the next lemma certain statements which were made in the introduction.

Lemma 2.5. The binary product $\alpha_{1} \circ \alpha_{2}$ is bilinear, associative and symmetric, and $\alpha_{1} \circ \alpha_{2} \circ \cdots \circ \alpha_{k}$ is equal to the $k$-fold product of $\alpha_{1}, \alpha_{2}, \cdots, \alpha_{k}$ in any order.

That the binary product $\alpha_{1} \circ \alpha_{2}$ is bilinear is clear; that it is associative follows from the equations

$$
\sum_{d} g_{c_{1} c_{2}}^{d} g_{d c_{3}}^{c}=\sum_{e} g_{c_{1} e}^{c} g_{c_{2} c_{3}}^{e}=g_{c_{1} c_{2} c_{3}}^{c}
$$

which results from counting the $g_{c_{1} c_{2} c_{8}}^{c}$ chains $V^{(0)}>V^{(1)}>V^{(2)}>0$ of submodules of $V^{(0)}=V_{c}$ which have factors isomorphic to $V_{c_{1}}, V_{c_{2}}, V_{c_{3}}$ respectively, in two ways: first we take all such chains for which $V^{(0)} / V^{(2)} \cong V_{d}$, and then sum over classes $d$ of $\mathcal{G}_{s_{1}+s_{2}}$; for the second way we sum, over classes $e$ of $\mathcal{S}_{s_{2}+s_{3}}$, the number of our chains in which $V^{(1)} \cong V_{\text {e }}$.

This formula shows in fact that $\alpha_{1} \circ\left(\alpha_{2} \circ \alpha_{3}\right)=\left(\alpha_{1} \circ \alpha_{2}\right) \circ \alpha_{3}=\alpha_{1} \circ \alpha_{2} \circ \alpha_{3}$, and similarly, we can show that $\alpha_{1} \circ \cdots \circ \alpha_{k}$ is equal to any $k$-fold product of $\alpha_{1}, \cdots, \alpha_{k}$ in that order. To complete the proof of Lemma 2.5, we must show that $\alpha_{1} \circ \alpha_{2}=\alpha_{2} \circ \alpha_{1}$. Let $A$ belong to the class $c$ of $\oiint_{s_{1}+s_{2}}$. It is well known that the transpose $A^{\prime}$ of any square matrix $A$ is similar to $A$, because $t 1-A$ and $t 1-A^{\prime}$ ( $t$ is an indeterminate) have the same elementary divisors. This means that $V_{A} \cong V_{A^{\prime}}$. But $V_{A^{\prime}}$ is isomorphic to the "dual module" $W_{A}$ of $V_{A}$, whose elements are the linear mappings of $V_{A}$ into $\mathfrak{F}$, and whose operator set consists of $\mathfrak{F}$ and the operator $A^{\prime}$ defined by

$$
\left(w A^{\prime}\right)(v)=w(v A) \quad\left(w \in W_{A}, v \in V_{A}\right)
$$

(we have used the same symbol $A^{\prime}$ for this linear transformation as for its matrix with respect to a suitable basis of $W_{A}$ ). Using the familiar correspondence between the subspaces of a vector space and its dual, we find that each chain

$$
V_{A}>V^{(1)}>0
$$

with factors isomorphic to $V_{c_{1}}, V_{c_{2}}$, respectively, corresponds to a chain

$$
0<W^{(1)}<W_{A}
$$

with factors again isomorphic to $V_{c_{1}}, V_{c_{2}}$. Thus $g_{c_{1} c_{2}}^{c}=g_{c_{2} c_{1}}^{c}$, and this proves that $\alpha_{1} \circ \alpha_{2}=\alpha_{2} \circ \alpha_{1}$.

P. Hall [7] has defined, for any partitions $\lambda_{1}, \lambda_{2}, \cdots, \lambda_{k}, \lambda$ such that $\left|\lambda_{1}\right|+\left|\lambda_{2}\right|+\cdots+\left|\lambda_{k}\right|=|\lambda|$, and for any prime-power $q$, the function $g_{\lambda_{1} \lambda_{2} \cdots \lambda_{k}}^{\lambda}(q)$, as the number of chains (3) of submodules of $V_{\lambda}$ in which the 
factor $V^{(i-1)} / V^{(i)}$ is isomorphic to $V_{\lambda_{i}}(i=1,2, \cdots, k)$. We shall make use of the following two theorems from this paper:

THEOREM 3. $g_{\lambda_{1} \lambda_{2} \cdots \lambda_{k}}^{\lambda}(q)$ is a polynomial in $q$, with rational integer coeffcients.

For a given partition $\lambda$, let $\{\lambda\}$ represent the Schur function (see Littlewood [9]) in an infinite (or any sufficiently large finite) number of variables $t_{1}, t_{2}, \cdots$. The product $\left\{\lambda_{1}\right\}\left\{\lambda_{2}\right\} \cdots\left\{\lambda_{k}\right\}$ can itself be written as a linear combination of Schur functions; let $c_{\lambda_{1} \lambda_{2}} \cdots \lambda_{k}$ be the coefficient of $\{\lambda\}$ in this expression. We have then

THEOREM 4. If $c_{\lambda_{1} \cdots \lambda_{k}}^{\lambda} \neq 0$, then $g_{\lambda_{1} \cdots \lambda_{k}}^{\lambda}(q)$ has the leading term $c_{\lambda_{1} \cdots \lambda_{k}}^{\lambda} q^{n \lambda-n \lambda_{1}-\cdots-n \lambda_{k}}$; but if $c_{\lambda_{1} \cdots \lambda_{k}}^{\lambda}=0$, then $g_{\lambda_{1} \cdots \lambda_{k}}^{\lambda}(q)$ vanishes identically. $\left(n_{\lambda}\right.$ is given by Definition 2.3.)

(We shall use from this theorem only the fact that the degree in $q$ of $g_{\lambda_{1} \cdots \lambda_{k}}^{\lambda}(q)$ is $n_{\lambda}-n_{\lambda_{1}}-\cdots-n_{\lambda_{k}}$, unless $g_{\lambda_{1} \cdots \lambda_{k}}^{\lambda}(q)$ vanishes identically.)

The following is a very brief sketch of the proofs of these results. Hall introduces for each partition $\lambda$ a symbol $\Gamma_{\lambda}$ and among these a multiplication

$$
\Gamma_{\lambda} \Gamma_{\mu}=\sum_{\nu} g_{\lambda \mu}^{\prime} \Gamma_{\nu}
$$

(It will be seen from the proof of Lemma 2.5 that it is enough to prove Theorems 3 and 4 for the $g_{\lambda_{\mu}}^{\nu}$.) If we write $E_{m}=\Gamma_{\{1 m\}}$, and $\Phi_{\lambda}=E_{k_{1}} E_{k_{2}} \cdots E_{k_{s}}$ (in the notation of Definitions 2.2 and 2.3), we find

$$
\Phi_{\lambda}=\Gamma_{\lambda}+\sum_{\mu} c_{\lambda \mu} \Gamma_{\mu}
$$

in which the $c_{\lambda \mu}$ are zero unless $\mu$ is a partition of $n=|\lambda|$ which precedes $\lambda$ in the usual ordering of partitions.

Next, the $c_{\lambda \mu}$ are polynomials in $q$ with rational integer coefficients; this is proved inductively by first calculating explicitly the coefficient $g_{\lambda,\{1 m\}}^{0}$ of $\Gamma_{\rho}$ in $\Gamma_{\lambda} E_{m}$ (for given partitions $\lambda, \rho$ and given integer $m$ ). This implies that the $\Gamma_{\lambda}$ can be expressed in terms of the $\Phi_{\mu}$ with coefficients which are polynomials in $q$. Theorem 3 now follows at once, for we have $\Phi_{\lambda} \Phi_{\mu}=\Phi_{k}$, where $\kappa$ is the partition whose conjugate has for its parts those of the conjugates of $\lambda$ and $\mu$ taken together.

Theorem 4 results from relating the possible structures of a module $V$ which has a chain (3) in which $V^{(i-1)} / V^{(i)}=V_{\lambda_{i}}(i=1,2, \cdots, k)$, with the law of multiplication of the Schur functions $\left\{\lambda_{1}\right\}, \cdots,\left\{\lambda_{k}\right\}$.

From Lemma 2.1 we have at once

Lemma 2.6. If $c=\left(\cdots f^{\nu(s)} \cdots\right)$, and if $c_{i}=\left(\cdots f^{\nu_{i}(f)} \cdots\right) \quad(i=1$, $2, \cdots, k)$, then 


$$
g_{c_{1} c_{2} \cdots c_{k}}^{c}=\prod_{f \in F} g_{\nu_{1}(f)}^{\nu(f)} \cdots \nu_{k}(f)\left(q^{d(f)}\right)
$$

We conclude by considering two special cases of (4). In the first of these (which comes most easily direct from Frobenius' formula $\psi^{*}(A)$ $\left.=\sum \psi\left(G_{i} A G_{i}^{-1}\right)\right), 1$ denotes the identity matrix of any appropriate degree.

LEMMA 2.7. If $\alpha_{1}, \cdots, \alpha_{k}$ are class-functions on $\mathbb{S}_{s_{1}}, \cdots, \mathfrak{S}_{s_{k}}$ respectively, and if $\alpha=\alpha_{1} \circ \cdots \circ \alpha_{k}$, then

$$
\alpha(1)=\frac{\psi_{s_{1}+\cdots+s_{k}}(q)}{\psi_{s_{1}}(q) \cdots \psi_{s_{k}}(q)} \alpha_{1}(1) \cdots \alpha_{k}(1),
$$

where $\psi_{n}(q)=\left(q^{n}-1\right)\left(q^{n-1}-1\right) \cdots(q-1)=(-1)^{n} \phi_{n}(q)$.

The proof follows from the easily verified facts, that $g_{n}=\psi_{n}(q) \cdot q^{C_{n, 2}}$ is the order of $\mathfrak{S}_{n}$, and that the order of $\mathfrak{S}_{s_{1}} \cdots_{s_{k}}$ is $g_{s_{1}} \cdots g_{s_{k}} \cdot q^{s}$, where

$$
s=\sum_{i<j} s_{i} s_{j}
$$

LeмmA 2.8. If $\alpha_{1}, \cdots, \alpha_{k}, \alpha$, are as in Lemma 2.7, and if $c=\left(f_{1} f_{2} \cdots f_{N}\right)$ is a principal class of $S_{n}$, then $\alpha(c)$ can be calculated from the values of $\alpha_{i}\left(c_{i}\right)$ at principal classes $c_{i}$ only; in fact

$$
\alpha(c)=\sum \alpha_{1}\left(c_{1}\right) \cdots \alpha_{k}\left(c_{k}\right)
$$

summed over those rows $\left(c_{1}, \cdots, c_{k}\right)$ of principal classes of $\mathbb{S}_{\mathbf{s}_{1}}, \cdots, \mathbb{S}_{\boldsymbol{s}_{k}}$, respectively, which are such that each of the polynomials $f_{1}, f_{2}, \cdots, f_{N}$ occurs in exactly one of the $c_{i}$.

For $V_{c}$ is the direct sum of characteristic submodules $V_{i}=V_{U\left(f_{i}\right)}(i=1$, $2, \cdots, N$ ) ; each $V_{i}$ is irreducible, and so the only submodules of $V_{c}$ are sums of subsets of $V_{1}, V_{2}, \cdots, V_{N}$. From this can be seen that each $g_{c_{1}}^{c} \cdots_{c_{k}}$ is either 0 or 1 , and is 1 only if $\left(c_{1} \cdots c_{k}\right)$ has the properties mentioned at the end of the lemma.

3. Brauer's theorem. The characters $I_{d}^{\boldsymbol{k}}[v]$. In this section we first prove the Theorem 1 stated in the introduction, and then construct with its help a large number of characters of the groups $\$_{n}$.

Brauer's Characterisation of Characters (Brauer [1]). A classfunction $\chi$ on a finite group $(S)$ is a character of $(B)$ if and only if the restriction of $\chi$ to $₹$ is a character of $\&$, for every elementary subgroup $\mathbb{F}$ of $(\$)$.

A subgroup $\&$ of $(S)$ is elementary if it is generated by an element $x \in(S)$, together with a Sylow $p$-subgroup $\mathfrak{P}$ of the centralizer of $x$ in $\mathfrak{B}, p$ being any prime which does not divide the order of $x$. Thus $\&$ is the direct product of $\mathfrak{B}$ with the cyclic subgroup generated by $x$.

Proof of theorem 1(4). We may assume that the homomorphism

(4) This theorem, and its proof, were suggested by one of Brauer's own applications of his characterisation of characters (Brauer [1, Theorem 12]). 


$$
\theta: \alpha \rightarrow \alpha_{0}
$$$$
\left(\alpha \in M\left(\mathfrak{F}^{*}\right)\right)
$$

is in fact any prescribed isomorphism of $M\left(\mathfrak{F}^{*}\right)$ into $M(\mathfrak{E})$. For otherwise, since $M\left(\mathfrak{F}^{*}\right)$ is cyclic, we have $\theta=\phi^{i}$ where $\phi$ is the prescribed isomorphism and $i$ is some integer. (By $\phi^{i}$ we understand here the function defined by $\phi^{i}(\alpha)=\phi\left(\alpha^{i}\right)$.) Then we have only to apply the theorem (supposed proved for $\phi)$ to the symmetric function $S\left(t_{1}^{4}, t_{2}^{1}, \cdots, t_{n}^{i}\right)$.

Suppose $\mathfrak{F}^{*}$ has characteristic $p$ and contains $p^{a}$ elements, and let $K$ be the algebraic number field obtained by adjoining the $\left(p^{a}-1\right)$ th roots of unity to the rationals. For any prime ideal divisor $p$ of $p$ in the ring $I$ of integers of $K$, we have $I / \mathfrak{p} \cong \mathfrak{F}^{*}$; let us identify $I / \mathfrak{p}$ with $\mathfrak{F}^{*}$. Let $R$ be the group of $\left(p^{a}-1\right)$ th roots of unity in $K$, then distinct elements of $R$ have distinct residues modulo $\mathfrak{p}$. Since we may choose the isomorphism $\theta$ arbitrarily, we assume that it is the inverse of the isomorphism $\kappa \rightarrow \bar{\kappa}=\kappa+p(\kappa \in R)$ of $R$ onto $M\left(\mathfrak{F}^{*}\right)$, so that $\overline{\alpha_{0}}=\alpha$ for each $\alpha \in M\left(\mathfrak{F}^{*}\right)$.

Our theorem is true if the order $g$ of $(S)$ is not divisible by the characteristic $p$ of $\mathfrak{F}^{*}$. For in this case there is a complex representation $R_{0}(x)$ of $\&$ with coefficients in $I$, such that

$$
\overline{R_{0}(x)}=R(x),
$$

all $x \in \mathbb{B}$

(see for example Speiser [11, p. 223]). The latent roots of $R_{0}(x)$ are complex $g$ th roots of unity $\kappa_{i}(x)$, which can be arranged so that

$$
\overline{\kappa_{i}(x)}=\xi_{i}(x) \quad(i=1,2, \cdots,) .
$$

On the other hand $\xi_{i}(x)_{0}$ is also a complex $g$ th root of unity such that

$$
\overline{\xi_{i}(x)_{0}}=\xi_{i}(x) \text {, }
$$

by our remarks above. Since $p$ does not divide $g$, no two distinct complex $g$ th roots of unity are congruent modulo $p$ (see for example, Brauer and Nesbitt, [2]), and therefore

$$
\xi_{i}(x)_{0}=\kappa_{i}(x) \quad(i=1,2, \cdots, n) .
$$

This shows that the $\xi_{i}(x)_{0}$ are the latent roots of a complex representation $R_{0}(x)$ of $\$ 5$, and it follows by a well-known theorem (Frobenius [4]) that any elementary symmetric function of the $\xi_{i}(x)_{0}$ is a (proper) character of $B$. This proves our theorem in the present case.

Suppose now that $(\mathbb{S}$ is an arbitrary finite group. Any elementary subgroup $(F$ of $\mathbb{B}$ can be written as a direct product $\mathfrak{S} \times \Omega$ of subgroups $\mathfrak{S}, \Omega$ of $\mathbb{S}$, where $\mathfrak{S}$ has order prime to $p$, and $\Omega$ is a $p$-group. By what we have proved, $\chi$ restricted to $\mathfrak{S}$ is a character of $\mathfrak{S}$. On the other hand, if $x \in \mathfrak{S}$ and $y \in \mathfrak{\Omega}$, then

$$
\chi(x y)=\chi(x) .
$$

For $R(y)$, whose order is a power of $p$, has all its latent roots equal to 1. $R(x)$ 
and $R(y)$ commute, so they can be transformed simultaneously to triangular matrices; we can assume that

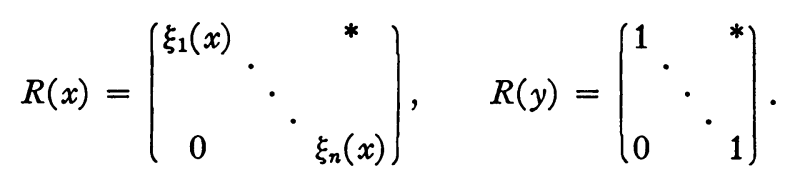

Thus $R(x y)=R(x) R(y)$ has latent roots $\xi_{1}(x), \cdots, \xi_{n}(x)$, which proves (5) (cf. Brauer and Nesbitt, loc. cit.).

Suppose for a moment that $\chi$ denotes an arbitrary proper character of $\mathfrak{S}$. We may extend $\chi$ to $\mathfrak{S} \times \Re$ by (5), and this extension is a character of $\mathfrak{S} \times \Re$, because $x y \rightarrow x$ is a homomorphic mapping of $\mathfrak{S} \times \Re$ onto $\mathfrak{E}$. By linearity this result applies to any character $\chi$ of $\mathfrak{H}$, and therefore to our present case. We have now shown that $\chi$ is a character on every elementary subgroup $\&$ of $(S)$, and so by Brauer's Theorem, $\chi$ is a character of $(S)$.

We take $R$ to be the defining representation $R(A)=A\left(A \in \mathcal{B}_{n}\right)$ of $\mathcal{B S}_{n}$, and $\mathfrak{F}^{*}=G F\left(q^{n !}\right)$ as in $\S 1$. Let $\theta$ be a fixed isomorphism of $M\left(\mathfrak{F}^{*}\right)$ into $M(\mathfrak{E}), \theta$ is a generator of the character group of the cyclic group $M\left(\mathfrak{F}^{*}\right)$, so that $\theta$ has multiplicative order $q^{n !}-1$. The restriction of $\theta$ to $M\left(\Im_{d}\right)(1 \leqq d \leqq n)$ is a generator of the character group of $M\left(\mathfrak{F}_{d}\right)$, and so $\theta$ has order $q^{d}-1$, as function on $M\left(\mathfrak{F}_{d}\right)$.

If $\alpha_{1}, \cdots, \alpha_{n}$ are the latent roots of $A \in B_{n}$, we have by Theorem 1 that

$$
\sigma_{r}^{k}(A)=\sum \theta^{k}\left(\alpha_{1}\right) \cdots \theta^{k}\left(\alpha_{r}\right),
$$

the $r$ th elementary symmetric function in $\theta^{k}\left(\alpha_{1}\right), \cdots, \theta^{k}\left(\alpha_{n}\right)$, is a character of $\mathfrak{S}_{n}$, for any integer $k$, and any positive integer $r \leqq n$. The case $r=n$ gives the linear character $\theta^{k}(\operatorname{det} A)$; we get $q-1$ different linear characters in this way, and it will later appear that these are all the linear characters of $\mathbb{S}_{n}$ (except in the case $n=q=2$ ).

Let us consider the value of the character $\sigma_{r}^{\boldsymbol{k}}$ at a principal class $c=\left(f_{1} f_{2} \cdots f_{N}\right)$ of $\mathcal{S}_{n} ; f_{1}, f_{2}, \cdots, f_{N}$ are distinct polynomials from $F$ whose degrees $d_{1}, d_{2}, \cdots, d_{N}$ add up to $n$. From each $f_{i}(i=1,2, \cdots, N)$ choose a single root $\gamma_{i}$, so that $\gamma_{i}^{\alpha^{u}}\left(u=0,1, \cdots, d_{i}-1\right)$ are all the roots of $f_{i}$. Write $t_{i}=\theta^{k}\left(\gamma_{i}\right)$ and $t_{i}^{a^{u}}=\theta^{k}\left(\gamma_{i}^{a^{u}}\right)$. We shall consider $t_{i}^{a^{u}}, t_{i}^{a^{\nu}}$ to be distinct "variables" if and only if $u \neq v\left(\bmod d_{i}\right)$; we have then a set of $n$ variables

$$
\begin{gathered}
t_{1}, t_{1}^{q}, t_{1}^{q^{2}}, \cdots, t_{1}^{q^{d_{1}-1}}, \\
t_{2}, t_{2}^{q}, t_{2}^{q^{2}}, \cdots, t_{2}^{q^{d_{2}-1}}, \\
\cdot \cdot \cdot \cdot \cdot \cdot \cdot \cdot, \\
t_{N}, t_{N}^{q}, t_{N}^{q^{2}}, \cdots, \underbrace{q^{d_{n-1}}}_{N} .
\end{gathered}
$$

By its definition, $\sigma_{r}^{\boldsymbol{k}}(c)=\sum m$, the sum of the $C_{n, r}$ monomials $m$ of degree $r$ in these variables, which contain each variable at most once. 
If $P$ is a set of permutations of the variables (6), and $m$ is a monomial, write $P m$ for the sum of the different monomials which can be obtained from $m$ by application of elements of $P$. Let $D_{i}$ be the cyclic group of order $d_{i}$ generated by the permutation which takes $t_{i}^{u^{u}}$ to $t_{i}^{\alpha^{u+1}}\left(u=0,1, \cdots, d_{i}-1\right)$ and leaves fixed the variables in the other rows of (6). Let $T$ be the subgroup generated by $D_{1}, \cdots, D_{N}$; it is the direct product of these groups.

If we collect the terms $m$ of $\sigma_{r}^{k}(c)=\sum m$ into sets of monomials $m$ transitive under $T$, we get

$$
\sigma_{r}^{k}(c)=\sum M,
$$

summed over all sums $M$ of such $T$-transitive sets. Each $M$ in (7) has the form $M=T m$, for some $m$, and if we write

$$
m=m_{1} m_{2} \cdots m_{N}
$$

$m_{i}$ being the product of the factors in $m$ which are from the $i$ th row of (6) $\left(m_{i}=1\right.$ if there are no such factors), it is clear that

$$
M=T m=\prod_{i=1}^{N} D_{i} m_{i} .
$$

To study a single factor $D_{i} m_{i}$ of (8) let us write for the moment $t_{i}=t$, $d_{i}=d, m_{i}=m$ and $D_{i}=D . m$ has the form

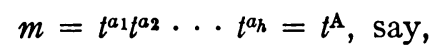

where A stands for the (unordered) set $\left(a_{1}, \cdots, a_{h}\right)=\left(q^{r_{1}}, \cdots, q^{r_{h}}\right)$ of $h \leqq r$ powers of $q$. Because $t^{q^{u}}=t^{q^{v}}$ if $u \equiv v(\bmod d)$ we think of $r_{1}, \cdots, r_{h}$ as residues $(\bmod d)$, and as such they are distinct. Such a set $\mathbf{A}$ of powers of $q$, in which we identify $q^{u}$ and $q^{v}$ if $u \equiv v(\bmod d)$, we call a $d$-set, and we say $\mathbf{A}$ is primitive if all the $d$-sets

$$
\mathrm{A}, \mathrm{A} q, \cdots, \mathrm{A} q^{d-1}
$$

are distinct. In general a $d$-set $\mathbf{A}$ is not primitive. Let $s$ be the least positive integer for which $\mathbf{A} q^{s}=\mathbf{A}$; then $s$ divides $d$ and there are exactly $s$ distinct sets (9), namely

$$
\mathrm{A}, \mathrm{A} q, \cdots, \mathrm{A} q^{s-1} \text {. }
$$

Because $\mathrm{A}=\mathrm{A} q^{s}$, we can collect the $h$ members of $\mathrm{A}$ into $g=h s / d$ subsets of the form

$$
\begin{aligned}
& q^{p_{1}}\left(1, q^{s}, \cdots, q^{s((d / s)-1)}\right), \\
& \left.q^{p_{o}\left(1, q^{s}\right.}, \cdots, q^{s((d / s)-1)}\right),
\end{aligned}
$$

and now $\mathbf{B}=\left(q^{p_{1}}, \cdots, q^{p_{\boldsymbol{\theta}}}\right)$ is a primitive $s$-set. 

let

Definition 3.1. If $s, e$ are positive integers, $k$ is any integer, and $\gamma \in F^{*}$,

$$
\begin{aligned}
T_{s, e}(k: \gamma)= & \theta^{k}\left(\gamma^{1+q^{8}+\ldots+q^{(\ell-1) s}}\right)+\theta^{q k}\left(\gamma^{1+q+\ldots+q^{(e-1) s}}\right) \\
& +\cdots+\theta^{q \sigma-1} k\left(\gamma^{1+q^{s}+\ldots+q^{(e-1) s}}\right) .
\end{aligned}
$$

This function, which is fundamental in the present work, will appear only in cases where the degree of $\gamma$ divides se (that is, when $\gamma \in M\left(\mathfrak{F}_{s e}\right)$ ). In this event $T_{s, e}(k: \gamma)=T_{s, e}\left(k: \gamma^{q}\right)$, so that $T_{s, e}(k: \gamma)$ is really a function of the irreducible polynomial $f$ which has $\gamma$ as a root. We can therefore write $T_{s, e}(k: \gamma)=T_{s, e}(k: f)$ without ambiguity.

We have from the definitions of $\mathrm{A}$ and $s$,

$$
D m=t^{\mathrm{A}}+t^{\mathrm{A} q}+\cdots+t^{\mathrm{A} q^{s-1}} .
$$

Taking $\mathbf{B}$ to be the primitive $s$-set derived from $\mathbf{A}$ above, and writing $k \mathrm{~B}=k\left(q^{p_{1}}+\cdots+q^{p_{\sigma}}\right)$, this gives

$$
D m=T_{s, d / 8}(k \mathrm{~B}: f),
$$

where $f=f_{i}$ is the polynomial from $\left(f_{1} f_{2} \cdots f_{N}\right)$ which is under consideration.

It is clear that $\mathbf{B}$ could be replaced by any of the sets $\mathrm{B} q, \mathbf{B} q^{2}, \ldots$ without altering the right-hand side of $(10)$. We shall call a pair $(s, \mathbf{B})$ in which

(i) $s$ is a positive integer, and

(ii) $\mathbf{B}$ is a primitive $s$-set, an index. Two indices $(s, \mathbf{B}),\left(s^{\prime}, \mathbf{B}^{\prime}\right)$ are considered equal if and only if $s=s^{\prime}$ and $\mathbf{B}=\mathbf{B}^{\prime} q^{u}$ for some integer $u$. The pair $(1,0)$, where 0 stands for the empty set, will be called the zero index. Except when this is expressly included, "index" will mean "nonzero index."

We have now from (8) and (10)

$$
M=\prod_{i=1}^{N} T_{s_{i}, d_{i} / s_{i}}\left(k \mathrm{~B}_{i}: f_{i}\right)
$$

where $\left(s_{i}, \mathbf{B}_{i}\right)$ is an index, possibly zero, such that $s_{i}$ divides $d_{i}$ $(i=1,2, \cdots, N)$. If $\left|\mathbf{B}_{i}\right|$ denotes the number of elements in $\mathbf{B}_{i}$, then $T_{s_{i}, d_{i} / s_{i}}\left(k \mathbf{B}_{i}: f_{i}\right)$ is of degree $d_{i}\left|\mathbf{B}_{i}\right| / s_{i}$ in the variables (6).

Let us define, for every pair $v, s$ of positive integers, and for every integer $k$, a function $I_{s}^{\boldsymbol{k}}[v]$ on the principal classes of $\mathfrak{S}_{s v}$, as follows. Let $\left(g_{1} g_{2} \cdots g_{M}\right)$ be a principal class of $\mathcal{B S}_{s v} ; g_{1}, g_{2}, \cdots, g_{M}$ being distinct polynomials from $F$. Then

$$
I_{s}^{k}[v]\left(g_{1} g_{2} \cdots g_{M}\right)=\prod_{i=1}^{M} T_{s, d\left(g_{i}\right) / s}\left(k: g_{i}\right)
$$

if $s$ divides $d\left(g_{i}\right)(i=1,2, \cdots, M)$, and is equal to zero otherwise. 
Let $\mathrm{c}(s, \mathbf{B})$ be the set of those $f_{i}$ occurring in $c=\left(f_{1} f_{2} \cdots f_{N}\right)$, which are such that $\left(s_{i}, \mathbf{B}_{i}\right)$ in (11) is a given index $(s, \mathbf{B})$, and write $v(s, \mathbf{B})=1 / s$ (sum of the degrees of these $\left.f_{i}\right), v(s, \mathbf{B})$ is an integer because each $s_{i}$ divides $d_{i}$, and if we use the symbol $\mathrm{c}(s, \mathrm{~B})$ to denote also the principal conjugacy class determined by the $f_{\boldsymbol{i}}$ which it contains, $\mathbf{c}(s, \mathbf{B})$ is a class of $\mathcal{G}_{s v(s, \mathbf{B})}$. We can now write

$$
M=\prod_{(s, \mathrm{~B})} I_{s}^{k \mathrm{~B}}[\mathrm{v}(s, \mathrm{~B})](\mathrm{c}(s, \mathrm{~B})),
$$

the product being taken over all (nonzero) indices $(s, \mathbf{B})$, with the convention that a factor for which $v(s, \mathbf{B})=0$ is equal to unity.

We have shown that every term $M$ of the sum

$$
\sigma_{r}^{k}(c)=\sum M
$$

determines the following pair of functions:

First, we have the non-negative integer-valued function $v$, defined for each index $(s, \mathrm{~B})$. This satisfies

$$
\sum_{(s, \mathrm{~B})} v(s, \mathrm{~B})|\mathrm{B}|=r,
$$

and

$$
\sum_{(\boldsymbol{s}, \mathrm{B})} s v(s, \mathrm{~B}) \leqq n ;
$$

the first equation expresses that each monomial in $M$ is of degree $r$ in the variables (6), while the left-hand side of the second is the sum of the degrees $d_{i}$ of those polynomials $f_{i}$ for which $\left(s_{i}, \mathbf{B}_{i}\right) \neq 0$, in (11).

Secondly we have the function $\mathbf{c}=c(s, \mathbf{B})$ whose values are subsets of $\left(f_{1} f_{2} \cdots f_{N}\right)$ (or, equivalently, the principal conjugacy classes which these subsets determine). For this we have

(C1) $\mathbf{c}(s, \mathbf{B})$ is a class of $\mathfrak{S}_{s v(s, \mathbf{B})}($ each $(s, \mathbf{B}))$,

(C2) $\mathrm{c}(s, \mathbf{B}), \mathbf{c}\left(s^{\prime}, \mathbf{B}^{\prime}\right)$ are disjoint unless $(s, \mathbf{B})=\left(s^{\prime}, \mathbf{B}^{\prime}\right)$, and

(C3) $s$ divides the degree of each member of $\mathbf{c}(s, \mathbf{B})$ (each $(s, \mathbf{B})$ ).

Conversely, such a pair of functions $\boldsymbol{v}$ and $\mathrm{c}$ determines, by means of (13), a term $M$ of (7).

Now suppose that for each $v$ satisfying (V1) and (V2), we define a classfunction $\Phi_{v}$ on the principal classes $c$ of $\mathcal{G}_{n}$ by

$$
\Phi_{v}=\left\{\prod_{(s, \mathrm{~B})} I_{*}^{k \mathrm{~B}}[v(s, \mathrm{~B})]\right\} \circ I_{m}
$$

where $\Pi$ denotes the o-product, $I_{m}$ is the identity character on $\mathcal{G}_{m}$, and

$$
m=n-\sum_{(s, \mathrm{~B})} s v(s, \mathrm{~B}) \text {. }
$$


By Lemma 2.8 this function is well defined on principal classes $c$, because we have defined the values of $I_{s}^{\mathbf{k}}[v]$ at principal classes.

The purpose of the foregoing analysis is the proof of the following

Lemma 3.2. For each principal class $c=\left(f_{1} f_{2} \cdots f_{N}\right)$ of $\mathbb{S}_{n}$,

$$
\left\{\sum \Phi_{\nabla}\right\}(c)=\sigma_{r}^{k}(c),
$$

the summation being over all functions $v$ which satisfy (V1) and (V2).

Proof. Lemma 2.8 shows that

$$
\Phi_{v}(c)=\sum_{c}\left\{\prod_{(s, \mathrm{~B})} I_{\bullet}^{k \mathrm{~B}}[v(s, \mathrm{~B})](\mathrm{c}(s, \mathrm{~B}))\right\},
$$

where the sum is over all functions c which satisfy (C1), (C2), and (C3). Thus the left-hand side of (15) is

$$
\sum_{v, \mathrm{O}}\left\{\prod_{(\boldsymbol{e}, \mathrm{B})} I_{\bullet}^{k \mathrm{~B}}[\mathrm{v}(s, \mathrm{~B})](\mathrm{c}(s, \mathrm{~B}))\right\},
$$

taken over all pairs $v, \mathrm{c}$ which satisfy (V1), (V2), (C1), (C2), (C3). By (13). this is exactly

$$
\sum M=\sigma_{r}^{k}(c) .
$$

We can now prove

THEOREM 5. Let $d, v$ be positive integers, and let $k$ be any integer. Then there is a character $I_{d}^{k}[v]$ of $\oiint_{d v}$ which has on the principal classes $c$ the value defined by (12).

Proof. This is by induction on the pair $(v, d)$. We shall say $(v, d)<\left(v^{\prime}, d^{\prime}\right)$ if either $v<v^{\prime}$, or $v=v^{\prime}$ and $d<d^{\prime}$. This is a well-ordering of the set of pairs $(v, d)$ of positive integers. For the first pair $(1,1)$ the theorem is true, for

$$
I_{1}^{k}[1](\alpha)=\theta^{k}(\alpha)
$$

is a character of $\mathfrak{G}_{1}=M(\mathfrak{F})$.

Take $n=d v, r=v$ in Lemma 3.2. Each $\Phi_{v}$ on the left-hand side of (15) is a o-product of $I_{z^{\prime}}^{\mathrm{kB}}\left[v^{\prime}\right]$ (together possibly with some identity character $I_{m}$ - see the definition (14) of $\Phi_{v}$ ) for which

$$
v^{\prime} \leqq v, \quad \text { and } \quad s^{\prime} v^{\prime} \leqq d v
$$

by (V1) and (V2) respectively. Therefore $\Phi_{v}$ has, as factors, only such $I_{\gamma^{\prime}}^{k^{\prime}}\left[v^{\prime}\right]$ as satisfy $\left(v^{\prime}, s^{\prime}\right) \leqq(v, d)$, and equality here can occur only if $\boldsymbol{v}$ is the function such that $v(s, \mathbf{B})=0$ unless $(s, \mathbf{B})=\left(d, q^{0}\right)$, while $v\left(d, q^{0}\right)=v$. In this case, $\Phi_{v}=I_{d}^{\mathbf{k}}[v]$. 
Therefore if we have found characters $I_{s^{\prime}}^{k^{\prime}}\left[v^{\prime}\right]$, for every $\left(v^{\prime}, s^{\prime}\right)<(v, d)$, which take on principal classes the values given by (12), we can use formula (15) (with $n=d v, r=v$ ) to extend the function $I_{d}^{k}[v]$ to the whole of $\mathcal{S}_{d v}$. Because the o-product of characters is again a character, and because $\sigma_{\boldsymbol{v}}^{\boldsymbol{k}}$ is a character, $I_{d}^{k}[v]$, so defined, is a character of $\mathfrak{S}_{d v}$.

EXAmple. Take $d=1$, and we are led to consider the equation (15) in the case $n=r=v$. Any function $v(s, \mathrm{~B})$ on the left must satisfy, by (V1) and (V2),

$$
\sum_{(s, \mathrm{~B})}|\mathrm{B}| \boldsymbol{v}(s, \mathrm{~B})=v, \quad \text { and } \quad \sum_{(s, \mathrm{~B})} s v(s, \mathrm{~B}) \leqq v .
$$

However, $\mathbf{B}$ is a primitive $s$-set, so that $|\mathbf{B}| \leqq s$, with equality only if $s=1$. Therefore $\boldsymbol{v}(s, \mathbf{B})=0$ unless $(s, \mathbf{B})=\left(1, q^{0}\right)$, and for this index we must have $v\left(1, q^{0}\right)=v$. Equation (15) now reads

$$
I_{1}^{k}[v]=\sigma_{v}^{k},
$$

and so we find that $I_{1}^{\mathbf{k}}[v]$ is one of the linear characters of $\left(\mathfrak{S}_{v}\right.$.

4. Uniform class functions. In this section and the next we are concerned to show that the characters $I_{d}^{\boldsymbol{k}}[v]$ are functions on $B_{d v}$ of a special sort, called uniform functions. One result of this is a rule for calculating the value $I_{d}^{k}[v](c)$ at any class $c$; another is that it becomes very easy to compute the scalar product of two such characters.

Definition 4.1. If $\lambda$ is a partition with $p \geqq 1$ parts, let $k(\lambda, q)=\phi_{p-1}(q)$ ( $\phi$ is given in Definition 2.2).

Definition 4.2. If $\lambda, \rho$ are partitions of $n$, and if $\rho=\left(1^{r_{1}} 2^{r_{2}} \cdots\right)$, let

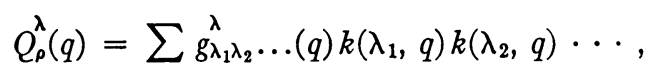

the sum being over all rows $\left(\lambda_{1}, \lambda_{2}, \cdots\right)$ of partitions, such that $\lambda_{1}, \cdots, \lambda_{r_{1}}$ are partitions of $1, \lambda_{r_{1}+1}, \cdots, \lambda_{r_{1}+r_{2}}$ are partitions of 2 etc.

There are close connections between these polynomials $Q_{\rho}^{\lambda}(q)$ and the characters $\chi_{\rho}^{\lambda}$ of the symmetric group $\widetilde{S}_{n}$, and in $\S 6$ we shall discover certain "orthogonality relations" among the $Q_{\rho}^{\lambda}$. For the moment we content ourselves with two elementary observations.

Lemma 4.3. The degree in $q$ of $Q_{\rho}^{\lambda}(q)$ does not exceed $n_{\lambda}$.

This follows from Hall's Theorem 4, together with the fact that the degree of $k(\nu, q)$ does not exceed $n_{\nu}$.

If $\rho=\left(1^{r_{1}} 2^{r_{2}} \cdots\right), \sigma=\left(1^{s_{1}} 2^{s_{2}} \cdots\right)$ are partitions of $l, m$ respectively, let $\rho+\sigma$ denote the partition $\left(1^{r_{1}+s_{1}} 2^{r_{2}+s_{2}} \cdots\right)$.

Lemma 4.4. If $\nu$ is any partition of $n$, then

$$
Q_{\rho+\sigma}^{\nu}=\sum g_{\lambda \mu}^{\nu} Q_{\rho}^{\lambda} Q_{\sigma}^{\mu},
$$

summed over pairs $\lambda, \mu$ of partitions respectively of $l, m$. 
Proof. Suppose that $\alpha_{1}, \alpha_{2}, \cdots$ and $\beta_{1}, \beta_{2}, \cdots$ are any two rows of partitions, such that $\left|\alpha_{1}\right|+\left|\alpha_{2}\right|+\cdots=l$, and $\left|\beta_{1}\right|+\left|\beta_{2}\right|+\cdots=m$. Then we have the identity

$$
\sum_{|\lambda|=l,|\mu|=m} g_{\lambda \mu}^{\prime}(q) g_{\alpha_{1} \alpha_{2}}^{\lambda} \ldots(q) g_{\beta_{1} \beta_{2}}^{\mu} \ldots(q)=g_{\alpha_{1} \alpha_{2} \ldots \beta_{1} \beta_{2}}^{\prime}(q) .
$$

For the right-hand side of (16) is the number of chains

$$
V>V^{(1)}>V^{(2)}>\cdots>W>W^{(1)}>W^{(2)}>\cdots>0
$$

of submodules of $V=V_{\nu}$ which have factors respectively isomorphic to $V_{\alpha_{1}}, V_{\alpha_{2}}, \cdots, V_{\beta_{1}}, V_{\beta_{2}}, \cdots$. The left-hand side is the sum for different pairs $\lambda, \mu$ of the number of chains (17) in which $V / W \cong V_{\lambda}, W \cong V_{\mu}$.

Thus

$$
\sum g_{\lambda \mu}^{\prime} Q_{p}^{\lambda} Q_{\sigma}^{\mu}=\sum g_{\alpha_{1} \alpha_{2}}^{\prime} \cdots \beta_{1} \beta_{2} \ldots(q) k\left(\alpha_{1}, q\right) k\left(\alpha_{2}, q\right) \cdots k\left(\beta_{1}, q\right) k\left(\beta_{2}, q\right) \cdots,
$$

and this equals $Q_{\rho+\sigma}^{\nu}$, in view of the fact that the Hall polynomial $g_{\lambda_{1} \lambda_{2}}^{\lambda} \ldots$ is symmetric in the suffixes $\lambda_{1}, \lambda_{2}, \cdots$ (this follows by induction from the formula $g_{c_{1} c_{2}}^{c}=g_{c_{2} c_{1}}^{c}$ proved in $\S 2$ ).

We may observe that Lemma 4.4 still holds if the $k(\lambda, q)$ are replaced by indeterminates $k_{\lambda}$. If $\rho=\left\{1^{r_{1}} 2^{r_{2}} \ldots\right\}$ is a partition of $n$, there is a principal type of class of $\mathfrak{B S}_{n}$, represented by

$$
c=\left(f_{11} \cdots f_{1 r_{1}} f_{21} \cdots f_{2 r_{2}} \cdots\right),
$$

$f_{d 1}, \cdots, f_{d r_{d}} \in F$ being distinct polynomials of degree $d(d=1,2, \cdots)$. We introduce a set $X^{\rho}$ of variables $x_{d i}^{\rho}=x_{d i}$, called $\rho$-variables. For each positive integer $d$ there are $r_{d}$ variables $x_{d 1}, \cdots, x_{d r_{d}}$, and each $x_{d i}$ is said to have degree $d\left(x_{d i}\right)=d\left(i=1,2, \cdots, r_{d}\right)$. Define a further set $\$ \rho$ of " $\rho$-roots"; these are $n$ variables, $d$ of which are called the roots of $x_{d i}$, and are written

$$
\xi_{d i}, \xi_{d i}^{q}, \cdots, \xi_{d i}^{q^{d-1}}
$$

$\left(i=1,2, \cdots, r_{d} ; d=1,2, \cdots\right)$. The $\rho$-roots are to be thought of as the latent roots of a "typical class"

$$
c=\left(x_{11} \cdots x_{1 r_{1}} x_{21} \cdots x_{2 r_{2}} \cdots\right) \text {. }
$$

Definition 4.5. A substitution of $X^{\rho}$ is a mapping $\alpha$ of $X^{\rho}$ into $F$ such that

$$
d(x \alpha) \text { divides } d(x) \text {, for each } x \in X^{\rho} .
$$

We apply $\alpha$ to $\Xi^{\rho}$ as well, by the following convention; for each $x_{d i} \in X^{\rho}$, choose any root $\gamma_{d i}$ of $x_{d i} \alpha$, and define $\xi_{d i}^{a} \alpha$ to be $\gamma_{d i}^{\alpha^{u}}(u=0,1, \cdots, d-1)$. (We shall use this notation only in contexts where it is irrelevant which root $\gamma_{d i}$ is chosen.)

Definition 4.6. Substitutions $\alpha, \alpha^{\prime}$ of $X^{\rho}$ are equivalent if there is a de- 
gree-preserving permutation $\phi$ of $X^{\rho}$ such that $\phi \alpha=\alpha^{\prime}$. A class of equivalent substitutions is called a mode of substitution.

Let $\alpha$ be a substitution of $X^{\rho}$, and let $f \in F$ have degree $d(f)=d$. For each positive integer $i$, let $r_{i}(\alpha, f)$ be the number of $x \in X^{p}$ such that $d(x)=i d$ and $x \alpha=f$. Then let $\rho(\alpha, f)$ be the partition

$$
\rho(\alpha, f)=\left\{1^{r_{1}(\alpha, f)} 2^{r_{2}(\alpha, f)} \cdots\right\} .
$$

We have immediately

Lemma 4.7. $\alpha, \alpha^{\prime}$ are equivalent substitutions of $X^{\rho}$ if and only if $\rho(\alpha, f)$ $=\rho\left(\alpha^{\prime}, f\right)$ for each $f \in F$.

Thus if $m$ is the mode of $\alpha$, we can write $\rho(\alpha, f)=\rho(m, f)$ without ambiguity.

It is clear that $|\rho(\alpha, f)| d(f)$ is the total degree of all the $\rho$-variables which are mapped into $f$ by $\alpha$, and consequently $\sum_{j \in F}|\rho(\alpha, f)| d(f)=|\rho|=n$. The converse of this is

Leмma 4.8. Let $\pi(f)$ be a partition-valued function on $F$ for which

$$
\sum_{f \in F}|\pi(f)| d(f)=n .
$$

Then there is a unique partition $\rho$ of $n$, and a unique mode $m$ of substitution of $X$, such that $\rho(m, f)=\pi(f)$, each $f \in F$.

Proof. Let $\pi(f)=\left\{1^{p_{1}(f)} 2^{p_{2}(f)} \cdots\right\}(f \in F)$. Define $\rho=\left\{1^{r_{1} 2^{r_{2}}} \cdots\right\}$ by

$$
r_{d}=\sum p_{i}(f)
$$

summed over the pairs $(i, f)$ ( $i$ is a positive integer, $f \in F)$ for which $i d(f)=d$ $(d=1,2, \cdots)$, and then $m$ is the mode of any substitution which takes, for each $f \in F$, exactly $p_{i}(f)$ of the $\rho$-variables of degree $i d(f)$ to $f$.

Definition 4.9. $A \rho$-function $U_{\rho}\left(x^{\rho}\right)=U_{\rho}\left(x_{11}, \cdots, x_{1 r_{1}} ; x_{21}, \cdots, x_{2 r_{2}}\right.$; ...) is a function in the $\rho$-variables which takes, for each substitution $\alpha$ of $X^{\rho}$, a complex value $U_{\rho}\left(x^{\rho} \alpha\right)=U_{\rho}\left(x_{11} \alpha, \cdots, x_{1 r_{1}} \alpha ; x_{21} \alpha, \cdots, x_{2 r_{2}} \alpha ; \cdots\right)$, and is symmetric in each set $x_{d 1}, \cdots, x_{d r d}(d=1,2, \cdots)$; in other words $U_{\rho}\left(x^{\rho} \alpha\right)=U_{\rho}\left(x^{\rho} \alpha^{\prime}\right)$ if $\alpha, \alpha^{\prime}$ are equivalent. We write $U_{\rho}\left(x^{\rho} \alpha\right)=U_{\rho}\left(x^{\rho} m\right)$, if $m$ is the mode of $\alpha$. $U_{\rho}$ is in fact just a complex-valued function on the set of modes $m$ of substitution of $X^{\rho}$.

In practice it is often convenient to express $U_{\rho}$ in terms of the $\rho$-roots. We shall write

$$
U_{\rho}\left(\xi^{\rho}\right)=U_{\rho}\left(\xi_{11}, \cdots, \xi_{1 r_{1}} ; \xi_{21}, \cdots, \xi_{2 r_{2}} ; \cdots\right)
$$

for $U_{\rho}\left(x^{\rho}\right)$, and, if $\gamma_{d i} \in M\left(\mathfrak{F}_{d}\right)$ is a root of $x_{d i} \alpha\left(i=1,2, \cdots, r_{d} ; d=1,2, \cdots\right)$, we define

$$
U_{\rho}\left(\xi^{\rho} \alpha\right)=U_{\rho}\left(\xi^{\rho} m\right)=U_{\rho}\left(\gamma_{11}, \cdots, \gamma_{1 r_{1}} ; \gamma_{21}, \cdots, \gamma_{2 r_{2}} ; \cdots\right)
$$


to be $U_{\rho}\left(x^{\rho} \alpha\right)$. Conversely if we have a function $U_{\rho}\left(\gamma_{11}, \cdots, \gamma_{1 r_{1}} ; \gamma_{21}, \cdots\right.$, $\left.\gamma_{2 r_{2}} ; \cdots\right)$ which takes a complex value whenever $\gamma_{d i} \in M\left(\mathfrak{F}_{d}\right)(i=1,2, \cdots$, $\left.r_{d} ; d=1,2, \cdots\right)$ is symmetric in each set $\gamma_{d 1}, \cdots, \gamma_{d r_{d}}(d=1,2, \cdots)$ and is unaltered if any $\gamma_{d i}$ is replaced by any conjugate, then the equation $U_{\rho}\left(\xi^{\rho} \alpha\right)=U_{\rho}\left(x^{\rho} \alpha\right)$ can be used to define $U_{\rho}\left(x^{\rho} \alpha\right)$ unambiguously, and we may regard $U_{\rho}$ as a $\rho$-function. Then

EXAMPLE. Let $\rho=\left\{2^{2}\right\}$. There are two $\rho$-variables $x_{21}, x_{22}$ each of degree 2 .

$$
\begin{aligned}
U_{\rho}\left(x_{21}, x_{22}\right) & =U_{\rho}\left(\xi_{21}, \xi_{22}\right)=T_{2,1}\left(k: \xi_{21}\right) T_{2,1}\left(k: \xi_{22}\right) \\
& =\left\{\theta^{k}\left(\xi_{21}\right)+\theta^{q k}\left(\xi_{21}\right)\right\}\left\{\theta^{k}\left(\xi_{22}\right)+\theta^{q k}\left(\xi_{22}\right)\right\}
\end{aligned}
$$

is a $\rho$-function, for clearly it gives a well-defined complex value $U_{\rho}\left(\xi_{21} \alpha, \xi_{22} \alpha\right)$ for each substitution $\alpha$, and equivalent substitutions give the same value.

Definition 4.10. Let $c=\left(\cdots f^{\nu_{c}(\gamma)} \cdots\right)$ be a class of $\oiint_{n}$, and let $\rho$ be a partition of $n$. Then a substitution of the $\rho$-variables into $c$ is a substitution $\alpha$ of $X^{\rho}$ which satisfies

$$
|\rho(\alpha, f)|=\left|\nu_{c}(f)\right| \quad(\operatorname{each} f \in F) .
$$

It is clear that if $m$ is a mode of substitution into $c$, it is equally a mode of substitution into $c^{\prime}$, where $c^{\prime}$ is any class for which

$$
\left.\left|\nu_{c^{\prime}}(f)\right|=\left|\nu_{c}(f)\right| \quad \text { (each } f \in F\right) .
$$

When this is the case we write $c \approx c^{\prime}$, and say that $c, c^{\prime}$ are isobaric. A given mode of substitution defines, by (S2), a unique set of isobaric classes.

Definition 4.11. If $\rho=\left\{1^{r_{1}} 2^{r_{2}} \cdots\right\}$, let $z_{\rho}=1^{r_{1} r_{1}} ! 2^{r_{2}} r_{2} ! \cdots$, and $w_{\rho}=r_{1} ! r_{2} ! \cdots$.

Definition 4.12 (Definition of UNIFORM FUnCtion). For each partition $\rho$ of $n$, let there be given a $\rho$-function $U_{\rho}\left(x^{\rho}\right)$. Then the uniform function $U=\left(U_{\rho}\right)$ on $\mathbb{S}_{n}$ is the class function defined at the class $c$ by

$$
U(c)=\sum_{\rho} \sum_{m} Q(m, c) U_{\rho}\left(x^{\rho} m\right)
$$

summed over partitions $\rho$ of $n$, and all modes $m$ of substitution of $X^{\rho}$ into $c$; and

$$
Q(m, c)=\prod_{f \in F} \frac{1}{z_{\rho(m, f)}} Q_{\rho(m, f)}^{r_{c}(f)}\left(q^{d(f)}\right) .
$$

The functions $U_{\rho}\left(x^{\rho}\right)$ are called the "principal parts" of $U, U_{\rho}$ being the " $\rho$-part." Formula (18) is called the degeneracy rule.

EXAMPLES. (1). Let $c=\left(f_{11} \cdots f_{1 r_{1}} f_{21} \cdots f_{2 r_{2}} \cdots\right)$ be a principal class of type $\rho=\left\{1^{r_{1}} 2^{r_{2}} \cdots\right\}$. There are no substitutions of $X^{\sigma}$ into $c$, unless $\sigma=\rho$; there is exactly one mode of substitution of $X^{\rho}$ into $c$, namely that represented by 


$$
x_{d i} \rightarrow f_{d i} \quad(i=1,2, \cdots, r ; d=1,2, \cdots) .
$$

Thus (18) gives $U(c)=U_{\rho}\left(f_{11}, \cdots, f_{1 r_{1}} ; f_{21}, \cdots, f_{2 r_{2}} ; \cdots\right)$. (2) Let $c$ be the class of the identity element of $\mathscr{B}_{n}$, so that $\nu_{c}(f)=0$ unless $f(t)=t-1$; while $\nu_{c}(t-1)=\left\{1^{n}\right\}$. The only mode of substitution of $X^{\rho}$ into $c$ is that represented by

$$
x_{d i} \rightarrow t-1 \quad\left(i=1,2, \cdots, r_{d} ; d=1,2, \cdots\right),
$$

and for this, $\rho(m, t-1)=\rho$. So we have

$$
U(1)=\sum_{|\rho|=n} \frac{1}{z_{\rho}} Q_{\rho}^{\left\{1^{n}\right\}}(q) U_{\rho}(1, \cdots, 1 ; 1, \cdots, 1 ; \cdots) .
$$

Definition 4.13. A uniform function $U$ whose principal parts are all zero except for $U_{\rho}$ is called a basic uniform function of type $\rho$.

We have at once

Lemma 4.14. Any uniform function $U$ is the sum $\sum_{\rho} U^{\rho}$ of basic uniform

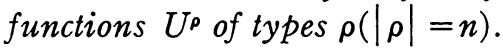

\section{Properties of uniform functions.}

THEOREM 6. If $U=\left(U_{p}\right), V=\left(V_{p}\right)$ are uniform functions on $\oiint_{n}$, and $\lambda, \mu$ are any complex numbers, then $\lambda U+\mu V$ is a uniform function on $\mathbb{S}_{n}$.

The proof is immediate, we take $\lambda U_{\rho}+\mu V_{\rho}$ as the $\rho$-part of $\lambda U+\mu V$.

THEOREM 7. If $U, V$ are uniform functions on $\mathbb{H}_{m}, \mathfrak{H}_{n}$ respectively, then $U \circ V$ is a uniform function on $\left(_{m+n}\right.$.

Proof. By Lemma 4.14 and Theorem 6 it is enough to show this for basic uniform functions $U, V$. Suppose that $U, V$ are basic of types $\rho=\left\{1^{r_{1}} 2^{r_{2}} \cdots\right\}$ and $\sigma=\left\{1 \cdot 2^{\circ 2} \ldots\right\}$ respectively, and that $U_{\rho}\left(x^{\rho}\right)$ is the $\rho$-part of $U$, and $V_{\sigma}\left(x^{\sigma}\right)$ is the $\sigma$-part of $V$. Write $\tau=\left\{1^{t_{1}} 2^{t_{2}} \ldots\right\}$ for the partition $\rho+\sigma$ of $m+n$.

A distribution $\pi$ will mean a 1-1 mapping of $X^{\rho} \cup X^{\sigma}$ onto $X^{\tau}$ which preserves the degree of each variable; $\pi, \pi^{\prime}$ are equivalent if $\phi \pi=\pi^{\prime}$ for some degree-preserving permutation $\phi$ of $X^{\rho} \cup X^{\sigma}$ which leaves each of the sets $X^{\rho}, X^{\sigma}$ invariant. An equivalence class $p$ of such distributions may be called a mode of distribution of $X^{\rho} \cup X^{\sigma}$ into $X^{\tau}$. We shall prove

Leмma 5.1. If $U, V$ are basic uniform functions of types $\rho, \sigma$ respectively, then $U \circ V$ is a basic uniform function of type $\tau=\rho+\sigma$ with $\tau$-part

$$
(U \circ V)_{\tau}\left(x^{\tau}\right)=\sum_{p} U_{\rho}\left(x^{\rho} p\right) V_{\sigma}\left(x^{\sigma} p\right),
$$

summed over all modes $p$ of distribution of $X^{\rho} \cup X^{\sigma}$ into $X^{\tau}$.

Thus for general $U=\left(U_{\rho}\right), V=\left(V_{\rho}\right)$, the $\tau$-part of $U \circ V$ is 


$$
(U \circ V)_{\tau}\left(x^{\tau}\right)=\sum_{\rho, \sigma, \rho+\sigma=\tau} \sum_{p} U_{\rho}\left(x^{\rho} p\right) V_{\sigma}\left(x^{\sigma} p\right) .
$$

Formula (20), less precisely described, is just Lemma 2.8 in the case $k=2$, $\alpha_{1}=U, \alpha_{2}=V$, with $c$ the "typical $\tau$-class"

$$
c=\left(x_{11}^{\tau} \cdots x_{1 t_{1}}^{\tau} x_{21}^{\tau} \cdots x_{2 t_{2}}^{\tau} \cdots\right) \text {. }
$$

Proof of Lemma 5.1. Let $c=\left(\cdots f^{(f)} \cdots\right)$ be a class of $\left(\mathfrak{S}_{m+n}\right.$. By Theorem 2 and the degeneracy rule (18),

$$
\begin{aligned}
(U \circ V)(c) & =\sum_{c_{1}, c_{2}} g_{c_{1} c_{2}}^{c} U\left(c_{1}\right) V\left(c_{2}\right) \\
& =\sum_{c_{1}, c_{2}} \sum_{m_{1}, m_{2}} g_{c_{1} c_{2}}^{c} Q\left(m_{1}, c_{1}\right) Q\left(m_{2}, c_{2}\right) U_{\rho}\left(x^{\rho} m_{1}\right) V_{\sigma}\left(x^{\sigma} m_{2}\right)
\end{aligned}
$$

summed over all classes $c_{1}, c_{2}$ of $\mathfrak{S}_{m}, \mathfrak{S}_{n}$ respectively, and all modes $m_{1}, m_{2}$ of substitutions of $X^{\rho}, X^{\sigma}$ respectively into $c_{1}, c_{2}$.

If $m_{1}$ is a mode of substitution of $X^{\rho}$ into $c_{1}$, it is equally a mode of substitution of $X^{\rho}$ into any class $c_{1}^{\prime}$ isobaric to $c_{1}$, and with this in mind we can write $(21)$ as

$$
(U \circ V)(c)=\sum_{m_{1}, m_{2}} R\left(m_{1}, m_{2}\right) U_{\rho}\left(x^{\rho} m_{1}\right) V_{\sigma}\left(x^{\sigma} m_{2}\right),
$$

summed over all pairs $m_{1}, m_{2}$ of modes of substitutions respectively of $X^{\rho}, X^{\sigma}$; and

$$
R\left(m_{1}, m_{2}\right)=\sum_{c_{1}^{\prime} \approx c_{1}, c_{2}^{\prime} \approx c_{2}} g_{c_{1}^{\prime} c_{2}^{\prime}}^{c} Q\left(m_{1}, c_{1}^{\prime}\right) Q\left(m_{2}, c_{2}^{\prime}\right)
$$

if $m_{1}, m_{2}$ are modes of substitutions into $c_{1}, c_{2}$ respectively.

Write $n_{i}(f)=\left|\nu_{c_{i}}(f)\right|(i=1,2 ; f \in F)$. By (19) and Lemma 2.4, we have

$$
\begin{aligned}
R\left(m_{1}, m_{2}\right)= & \prod_{f \in F} \sum_{\left|\nu_{1}\right|=n_{1}(f),\left|\nu_{2}\right|=n_{2}(f)} g_{\nu_{1} \nu_{2}}^{\nu(f)}\left(q^{d(f)}\right) \\
& \cdot \frac{1}{z_{\rho\left(m_{1}, f\right)} z_{\sigma\left(m_{2}, f\right)}} Q_{\rho\left(m_{1}, f\right)}^{\nu_{1}}\left(q^{d(f)}\right) Q_{\sigma\left(m_{2}, f\right)}^{\nu_{2}}\left(q^{d(f)}\right) .
\end{aligned}
$$

What we require to show is that $(U \circ V)(c)$ is equal to

$$
\sum_{m} Q(m, c)(U \circ V)_{\tau}\left(x^{\tau} m\right)=\sum_{m} \sum_{p} Q(m, c) U_{\rho}\left(x^{\rho} p m\right) V_{\sigma}\left(x^{\sigma} p m\right)=Y, \text { say, }
$$

the last summation being over modes $m$ of substitution of $X^{\tau}$ into $c$, and over modes $p$ of distribution of $X^{\rho} \cup X^{\sigma}$ into $X^{\tau}$.

If $\alpha, \alpha_{1}, \alpha_{2}$ are substitutions of the respective modes $m, m_{1}, m_{2}$, then there exists a distribution $\pi$ such that 


$$
\begin{aligned}
& \alpha_{1}=\pi \alpha\left(\text { restricted to } X^{\rho}\right), \text { and } \\
& \alpha_{2}=\pi \alpha\left(\text { restricted to } X^{\sigma}\right),
\end{aligned}
$$

if and only if

$$
\rho\left(m_{1}, f\right)+\sigma\left(m_{2}, f\right)=\tau(m, f) \quad(\text { each } f \in F) .
$$

With given $m_{1}$ and $m_{2}$, equations (25) determine $m$ (Lemma 4.8), and the number of modes $p$ of distributions $\pi$ which satisfy (24) is easily found to be

$$
S\left(m_{1}, m_{2}\right)=\prod_{f \in F} \frac{w_{\tau(m, f)}}{w_{\rho\left(m_{1}, f\right)} w_{\sigma\left(m_{2}, f\right)}}
$$

( $w_{\rho}$ is given in Definition 4.11). However this number is the number of terms $Q(m, c) U_{\rho}\left(x^{\rho} p m\right) V_{\sigma}\left(x^{\sigma} p m\right)$ in $Y$, for which $U_{\rho}\left(x^{\rho} p m\right)=U_{\rho}\left(x^{\rho} m_{1}\right)$, and $V_{\sigma}\left(x^{\sigma} p m\right)$ $=V_{\sigma}\left(x^{\sigma} m_{2}\right)$, so that the coefficient in $Y$ of $U_{\rho}\left(x^{\rho} m_{1}\right) V_{\sigma}\left(x^{\sigma} m_{2}\right)$ is

$$
S\left(m_{1}, m_{2}\right) Q(m, c)=T\left(m_{1}, m_{2}\right) \text {, say. }
$$

If we apply Lemma 4.4, together with (25), to the expression (23) for $R\left(m_{1}, m_{2}\right)$ we find with little difficulty that $T\left(m_{1}, m_{2}\right)$ is the same as $R\left(m_{1}, m_{2}\right)$. Thus comparing $Y$ with (23) we have $Y=(U \circ V)(c)$, and the lemma is proved.

As far as the last two theorems are concerned, the nature of the $k(\lambda, q)$ which appear in the definition of $Q_{\rho}^{\lambda}(q)$ is irrelevant; in Lemma 4.4 we could replace the $k(\lambda, q)$ by indeterminates $k_{\lambda}$. Our next theorem, however, which is the link between uniform functions and the characters $I_{d}^{k}[v]$ obtained in $\S 3$, depends essentially on the definition (4.1) $k(\lambda, q)=\phi_{p-1}(q)$.

Theorem, 8. Let $S\left(\gamma_{1}, \gamma_{2}, \cdots, \gamma_{n}\right)$ be a function symmetric in $\gamma_{1}, \gamma_{2}, \cdots$, $\gamma_{n}$ which takes complex values whenever $\gamma_{1}, \gamma_{2}, \cdots, \gamma_{n} \in M\left(\mathfrak{F}^{*}\right)$. Then the classfunction $S(c)$ on $\mathbb{S}_{n}$, whose value at a class $c$ with latent roots $\gamma_{1}, \gamma_{2}, \cdots, \gamma_{n}$ is defined to be

$$
S(c)=S\left(\gamma_{1}, \gamma_{2}, \cdots, \gamma_{n}\right),
$$

is a uniform function on $\mathcal{B}_{n}$, with principal $\rho$-part

$$
S_{\rho}\left(x^{\rho}\right)=S\left(\xi_{1}^{\rho}, \xi_{2}^{\rho}, \cdots, \xi_{n}^{\rho}\right),
$$

where $\xi_{1}^{\rho}, \xi_{2}^{\rho}, \cdots, \xi_{n}^{\rho}$ are the $\rho$-roots in any order ( $\rho$ is any partition of $n$ ).

Proof. We have to show that for the class $c=\left(\cdots f^{\prime(f)} \cdots\right)$,

$$
S\left(\gamma_{1}, \cdots, \gamma_{n}\right)=\sum_{\rho} \sum_{m} Q(m, c) S_{\rho}\left(\xi^{\rho} m\right),
$$

$\gamma_{1}, \cdots, \gamma_{n}$ being the latent roots of $c$. By the definition of $S_{\rho}\left(x^{\rho}\right)$,

$$
S_{\rho}\left(\xi^{\rho} m\right)=S\left(\xi_{1}^{\rho} \alpha, \cdots, \xi_{n}^{\rho} \alpha\right)=S\left(\gamma_{1}, \cdots, \gamma_{n}\right),
$$


$\alpha$ being any substitution of a mode $m$, and $m$ being a mode of substitution of $X^{\rho}$ into $c$. This holds for every partition $\rho$ of $n$. Thus it is enough to show that

$$
\sum_{\rho, m} Q(m, c)=1,
$$

where the summation is over all partitions $\rho$ of $n$, and all modes $m$ of substitution of $X^{\rho}$ into $c$. By (19)

$$
\begin{aligned}
\sum_{\rho, m} Q(m, c) & =\sum_{\rho, m} \prod_{f \in F} \frac{1}{z_{\rho(m, f)}} Q_{\rho(m, f)}^{\nu(f)}\left(q^{d(f)}\right) \\
& =\prod_{f \in F}\left\{\sum_{|\pi|=|\nu(f)|} \frac{1}{z_{\pi}} Q_{\pi}^{\nu(f)}\left(q^{d(f)}\right)\right\},
\end{aligned}
$$

because as $\rho, m$ take all the values we have mentioned, the function $\rho(m, f)$ runs over precisely the set of partition-valued functions $\pi(f)$ such that $|\pi(f)|=|\nu(f)|(f \in F)$, by Lemma 4.8. Therefore (26) follows from

Lemma 5.2. Let $\nu$ be a partition of $n$. Then

$$
\sum_{|\rho|=n} \frac{1}{z_{\rho}} Q_{p}^{\prime}(q)=1 .
$$

Proof. This is by induction on $n$. If $\tau=\left\{1^{t_{1}} 2^{t_{2}} \ldots\right\}$ is a partition of $n$, if $1 \leqq s \leqq n$, and if $t_{s} \geqq 1$, let $\tau^{*}$ be the partition $\left\{1^{t_{1}} \cdots s^{t_{2}-1} \cdots\right\}$ of $n-s$. Any partition $\rho$ of $n-s$ can be written uniquely in the form $\rho=\tau^{*}$, for some partition $\tau$ of $n$.

By Lemma 4.4,

$$
Q_{\tau}^{\prime}=\sum_{|\lambda|-n-s,|\mu|-s} g_{\lambda_{\mu}}^{\prime} Q_{\tau}^{\lambda} \cdot Q_{s}^{\mu},
$$

and so, observing that $z_{\tau}=s t_{s} z_{\tau^{*}}$,

$$
\sum_{\lambda, \mu} g_{\lambda_{\mu}}^{\nu} \frac{1}{z_{\tau^{*}}} Q_{\tau^{*}}^{\lambda} Q_{\varepsilon}^{\mu}=\frac{1}{z_{\tau}} \cdot s t_{\bullet} \cdot Q_{\tau}^{\nu}
$$

if $\tau^{*}$ exists, that is, if $t_{8}>0$. If $t_{s}=0, \tau^{*}$ does not exist, but in this case the right-hand side is zero, so we have, summing over all partitions $\rho\left(=\tau^{*}\right)$ of $n-s$,

$$
\sum_{|\rho|=n-\varepsilon} \sum_{\lambda, \mu} g_{\lambda_{\mu}}^{\nu} \frac{1}{z_{\rho}} Q_{\rho}^{\lambda} Q_{s}^{\mu}=\sum_{|\tau|=n} \frac{1}{z_{\tau}} Q_{\tau}^{\nu} \cdot s t_{s} .
$$

By the induction hypothesis, $\sum_{\rho}\left(1 / z_{\rho}\right) Q_{\rho}^{\lambda}=1$ if $s \geqq 1$, therefore

$$
\sum_{\lambda, \mu} g_{\lambda \mu}^{\prime} Q_{s}^{\mu}=\sum_{|\tau|=n} \frac{1}{z_{\tau}} Q_{\tau}^{\prime} \cdot s t_{s} \quad(s=1,2, \cdots, n) .
$$

Define now polynomials $h_{\mu}^{p}=h_{\mu}^{\nu}(q)$ by 


$$
h_{\mu}^{\prime}=\sum_{|\lambda|=n-s} g_{\lambda \mu}^{\prime} \quad(|\nu|=n,|\mu|=s),
$$

and then summing the preceding equation over $s=1,2, \cdots, n$, we have

$$
\sum_{0<|\mu| \leqq_{n}} h_{\mu}^{\prime \prime}(q) k(\mu, q)=\left\{\sum_{|\tau|=n} \frac{1}{z_{\tau}} Q_{\tau}^{\nu}\right\} \sum_{s} s t_{s}=n\left\{\sum_{\tau} \frac{1}{z_{\tau}} Q_{\tau}^{\nu}\right\} .
$$

The proof of this lemma, and hence of Theorem 8 , now rests on the following final result:

Lemma 5.3. If $\nu$ is a partition of $n$, and if $h_{\mu}^{\nu}(q)$ are the polynomials defined in (27), then

$$
\sum_{0<|\mu| \leqq n} h_{\mu}^{\prime}(q) k(\mu, q)=n .
$$

Proof. $h_{\mu}^{\nu}(q)$ is by Definition (27) the number of submodules of $V_{\nu}$ (see $\S 2$ ) which are isomorphic to $V_{\mu}$. It is clear that every submodule $S$ of $V_{\nu}$ is isomorphic to some $V_{\mu}$, where $|\mu| \leqq n$. Let us define for submodules $S$ of $V$, the functions

$$
\begin{aligned}
& f(S)=k(\mu, q), \text { if } S \cong V_{\mu}(f(0)=0), \text { and } \\
& g(S)=\text { dimension of } S \text { as vector space over } \mathfrak{F} .
\end{aligned}
$$

Then equation (28) says

$$
g\left(V_{v}\right)=\sum_{S} f(S)
$$

summed over all submodules $S$ of $V_{\nu}$. If $|\nu|=1$, the only submodules of $V_{\nu}$ are $V_{\nu}$ and 0 , so that the formula holds in this case. We shall prove (29) by induction on $|\nu|=g\left(V_{\nu}\right)$. To this end we quote an easy adaptation of P. Hall's enumeration formula for p-groups (Hall [6]), as follows. Write $U$ for the matrix $U_{\nu}(t)$ (see $\left.\S 1\right) ; V_{\nu}$ is by definition the module whose operators are $U$ and the field $\mathfrak{F}$. The submodule $D=V_{\nu} U$ is the intersection of the maximal submodules of $V_{\nu}$, and $V_{\nu} / D$ has $\mathfrak{F}$-dimension $d=$ number of parts of the partition $\nu$. Further, since $v U \equiv 0(\bmod D)$ for every $v \in V_{\nu}$, we have that any F-subspace of $V_{v} / D$ is a submodule. It is easy to see that the number of subspaces of $V_{v} / D$ which have dimension $d-k(0 \leqq k \leqq d)$ is

$$
N_{k}^{d}=N_{k}^{d}(q)=\frac{\left(q^{d}-1\right)\left(q^{d-1}-1\right) \cdots\left(q^{d-k+1}-1\right)}{\left(q^{k}-1\right)\left(q^{k-1}-1\right) \cdots(q-1)} .
$$

Hall's enumeration formula (Hall, loc. cit., Theorem 1.4, p. 39) says that if $f(S)$ is any function defined on the submodules $S$ of $V_{v}$, and for each submodule $T$ we define $g(T)$ to be $\sum f(S)$, summed over all submodules $S \subseteq T$, then

$$
\begin{aligned}
g\left(V_{v}\right)= & f\left(V_{v}\right)+\sum g\left(S_{1}\right)-q \sum g\left(S_{2}\right)+\cdots+(-1)^{k-1} q^{C_{k, 2}} \sum g\left(S_{k}\right) \\
& +\cdots+(-1)^{d-1} q^{C_{d, 2}} \sum g\left(S_{d}\right),
\end{aligned}
$$


where in $\sum g\left(S_{k}\right)$ the summation is over all submodules $S_{k}$ containing $D$, for which the dimension of $S_{k} / D$ is $d-k$ (and for which, therefore, the dimension of $S_{k}$ is $n-k$ ). This formula (which is based on the identity (30) below) can be proved in just the same way as the corresponding formula for $p$-groups.

In our case, $\sum g\left(S_{k}\right)=(n-k) N_{k}^{d}$, for by the induction hypothesis, $g\left(S_{k}\right)$ is equal to the $\mathfrak{F}$-dimension of $S_{k}$, provided that this is less than $n$; that is, if $k \geqq 1$. Therefore

$$
\begin{aligned}
g\left(V_{\nu}\right) & =k(\nu, q)+\sum_{k=1}^{d}(n-k) N_{k}^{d}(-1)^{k-1} q^{C_{k, 2}} \\
& =k(\nu, q)+n \sum_{k=1}^{d}(-1)^{k-1} q^{C_{k, 2}} N_{k}^{d}-\sum_{k=1}^{d}(-1)^{k-1} k q^{C_{k, 2}} N_{k .}^{d} .
\end{aligned}
$$

We have however the identity (Hall, loc. cit.)

$$
\sum_{k=0}^{d} q^{C_{k, 2}} N_{k}^{d} t^{k}=(1+t)(1+q t) \cdots\left(1+q^{d-1} t\right)
$$

Putting $t=-1$ in this gives

$$
\sum_{k=1}^{d}(-1)^{k-1} q^{C_{k, 2}} N_{k}^{d}=1,
$$

while if we differentiate with respect to $t$, and then put $t=-1$, we find

$$
\sum_{k=1}^{d}(-1)^{k-1} k q^{C_{k, 2}} N_{k}^{d}=\phi_{d-1}(q) .
$$

Thus $g\left(V_{\nu}\right)=k(\nu, q)+n-\phi_{d-1}(q)=n$, which completes the proof of Lemma 5.3.

THEOREM 9. For given positive integers $d$ and $v$, and a given integer $k$, the character $I_{d}^{k}[v]$ is the uniform function $I_{d}^{k}[v]=U$ on $\oiint_{d v}$ with the following principal parts:

$U_{\rho}=0$ unless all the parts of $\rho$ are divisible by $d$, while if $\rho=\left\{d^{p_{1}}(2 d)^{p_{2}} \cdots\right\}$,

$$
U_{\rho}\left(x^{\rho}\right)=U_{\rho}\left(\xi^{\rho}\right)=\prod_{e} \prod_{i=1}^{p_{e}} T_{d, e}\left(k: \xi_{e d, i}\right),
$$

where

$$
T_{d, e}(k ; \xi)=\sum_{i=0}^{d-1} \theta^{i k k}\left(\xi^{1+q^{d}+\cdots+q^{(\sigma-1) d}}\right) .
$$

Proof. Let $\rho=\left\{1^{r_{1}}, 2^{r_{2}} \ldots\right\}$ be a partition of $n$, and let $c$ stand for a "typical $\rho$-class" $\left(x_{11} \cdots x_{1 r_{1}} x_{21} \cdots x_{2 r_{2}} \cdots\right)$. Define $\sigma_{r}^{k}(c)$ to be $\sum \theta^{k}\left(\xi_{1}^{p}\right)$ $\cdots \theta^{k}\left(\xi_{\tau}^{\rho}\right)$, where $\xi_{1}^{\rho}, \cdots, \xi_{n}^{\rho}$ is the set $\Xi^{\rho}$ of $\rho$-roots, in some order. This purely formal definition makes the principal $\rho$-part of the function $\sigma_{\tau}^{k}$ (which 
is known to be uniform, by Theorem 8) the "value" of $\sigma_{r}^{k}$ at the "class" $c$. It is easy to verify that Lemma 3.2, which is the result of an analysis of the value of $\sigma_{r}^{k}(c)$ at a principal class $c=\left(f_{1} f_{2} \cdots f_{N}\right)$, will still hold for this "typical $\rho$-class" $c$. The only changes needed are to replace, in the argument leading to equation (13), the latent roots $\gamma_{i}^{a^{*}}\left(u=0,1, \cdots, d_{i}-1 ; i=1,2\right.$, $\cdots, N)$ by the $\rho$-roots, and to interpret each $I_{s}^{\mathrm{kB}}[\boldsymbol{v}(s, \mathrm{~B})]$ which occurs in (14) as the uniform function defined in the present theorem. By Theorem 7 the function $\Phi_{\nabla}$ so defined is uniform, and its $\rho$-part is the result of evaluating $\Phi_{v}(c)$ formally by Lemma 2.8 (see equation (20), Theorem 7 ). With these interpretations the proof of Lemma 3.2 still holds, and (15) is now a relation between the principal $\rho$-parts of the uniform functions $\Phi_{v}$ and $\sigma_{r}^{k}$.

The character $I_{d}^{\mathbf{k}}[v]$ is defined, in the proof of Theorem 5 , inductively with the help of (15). We have shown that the same relation (15) holds between the uniform functions $I_{d}^{\boldsymbol{k}}[v]$ defined in Theorem 9. Therefore we can prove, inductively, that the character and uniform function coincide, and this is the assertion of Theorem 9.

The degeneracy rule (18) allows us to calculate, in terms of the polynomials $Q_{\rho}^{\lambda}$, the values of these characters $I_{d}^{k}[v]$ at any class.

EXAMPLE (i). $U=I_{2}^{k}[2]$ is a character of $\mathcal{S}_{4}$. We have that $U_{\rho}=0$ for all partitions $\rho$ of 4 except $\rho=\left\{2^{2}\right\}$ and $\rho=\{4\}$. Let $x_{21}, x_{22}$ be the $\left\{2^{2}\right\}$-variables, and let $x_{41}$ be the $\{4\}$-variable (in our general notation these sets of variables would be distinguished by superscripts $\left\{2^{2}\right\}$ and $\{4\}$ respectively). Then

$$
\begin{aligned}
U_{\left\{2^{2}\right\}}\left(x_{21}, x_{22}\right) & =\left\{\theta^{k}\left(\xi_{21}\right)+\theta^{q k}\left(\xi_{21}\right)\right\}\left\{\theta^{k}\left(\xi_{22}\right)+\theta^{q k}\left(\xi_{22}\right)\right\}, \text { and } \\
U_{\{4\}}\left(x_{41}\right) & =\theta^{k}\left(\xi_{41}^{1+q^{2}}\right)+\theta^{q k}\left(\xi_{41}^{1+q^{2}}\right) .
\end{aligned}
$$

Let $c=\left(f^{\left.\left(1^{2}\right\}\right)}\right.$, where $f \in F$ has degree 2 ; this is a class of $\mathbb{B}_{4}$. In each of the cases $\rho=\left\{2^{2}\right\}$ and $\rho=\{4\}$, there is only one mode of substitution of $X^{\rho}$ into $c$; these are the modes $m_{1}, m_{2}$ of the substitutions

$$
x_{21}, x_{22} \rightarrow f \text {, and } x_{41} \rightarrow f,
$$

respectively. Writing $\gamma$ for a root of $f$, we have

$$
\begin{aligned}
U(c) & =Q\left(m_{1}, c\right) U_{\left\{2^{2}\right\}}(\gamma, \gamma)+Q\left(m_{2}, c\right) U_{\{4\}}(\gamma) \\
& =2^{-1}\left(q^{2}+1\right)\left\{\theta^{k}(\gamma)+\theta^{a k}(\gamma)\right\}^{2}+2^{-1}\left(-q^{2}+1\right)\left\{\theta^{k}\left(\gamma^{2}\right)+\theta^{a k}\left(\gamma^{2}\right)\right\} \\
& =\theta^{k}\left(\gamma^{2}\right)+\theta^{a k}\left(\gamma^{2}\right)+\left(q^{2}+1\right) \theta^{k}(\gamma) \theta^{a k}(\gamma) .
\end{aligned}
$$

EXAMPLE (ii). Let $U=I_{d}^{k}[1]$, a character of $\mathfrak{S}_{d}$. We have $U_{\rho}=0$, unless $\rho=\{d\}$. There is only one $\{d\}$-variable, $x_{d 1}$, and $U_{\{d\}}\left(\xi_{d 1}\right)=T_{d, 1}\left(k: \xi_{d 1}\right)$. The only classes $c$ fo- which there exist substitutions of $X^{\{d\}}$ into $c$ are the primary classes $c=\left(f^{\lambda}\right)$, with $|\lambda| d(f)=d$. Write $e=d / d(f)$, and let $\gamma$ be a root of $f$. The only mode $m$ is that of the substitution $x_{d 1} \rightarrow f$, and for this, $\rho(m, f)=\{e\}$. Therefore 


$$
U(c)=Q(m, c) T_{d, 1}(k: \gamma)=\frac{1}{e} Q_{(d)\}}^{\lambda}\left(q^{d(f)}\right) T_{d, 1}(k: \gamma) .
$$

It is easy to see from Definition 4.2 that $Q_{\{\ell\}}^{\lambda}(q)=k(\lambda, q)$; while

$$
T_{d, 1}(k: \gamma)=\sum_{i=0}^{d-1} \theta^{q^{i k} k}(\gamma)=e \sum_{i=0}^{d(f)-1} \theta^{q^{i k} k}(\gamma),
$$

because $\gamma$ has degree $d(f)$. Thus we have $I_{d}^{k}[1](c)=0$ unless $c$ is primary, while

$$
I_{d}^{k}[1]\left(f^{\lambda}\right)=k\left(\lambda, q^{d(f)}\right)\left\{\theta^{k}(\gamma)+\theta^{q k}(\gamma)+\cdots+\theta^{q^{d(f)-1} k}(\gamma)\right\},
$$

where $\gamma$ is any root of the polynomial $f$.

6. Scalar product of uniform functions. If $U, V$ are any complex-valued class-functions on $\mathcal{G H}_{n}$, the scalar product

$$
(U, V)=\frac{1}{g} \sum_{A \in \Theta_{n}} U(A) \bar{V}(A)=\sum_{c} \frac{1}{a(c)} U(c) \bar{V}(c),
$$

summed over classes $c$ of $\oiint_{n}$. For $g / a(c)$ (see $\$ 2$ ) is the number of elements in the class $c$. If $c=\left(\cdots f^{v(f)} \cdots\right)$ we have by Lemma 2.4

$$
a(c)=\sum_{f \in F} a_{\nu(f)}\left(q^{d(f)}\right) .
$$

We shall find that when $U, V$ are uniform functions, $(U, V)$ has a very simple expression (Theorem 11). First however we shall write down certain remarkable "orthogonality relations" satisfied by the polynomials $Q_{\rho}^{\lambda}$.

Definition 6.1. If $\rho=\left\{1^{r_{1}} 2^{r_{2}} \ldots\right\}$, let

$$
c_{\rho}(q)=(q-1)^{r_{1}}\left(q^{2}-1\right)^{r_{2}} \cdots .
$$

THEOREM 10. If $\rho, \sigma$ are partitions of $n$, then

$$
\sum_{|\lambda|=n} \frac{1}{a_{\lambda}(q)} Q_{\rho}^{\lambda}(q) Q_{\sigma}^{\lambda}(q)= \begin{cases}0 & \text { if } \rho \neq \sigma, \\ \frac{z_{\rho}}{c_{\rho}(q)} & \text { if } \rho=\sigma .\end{cases}
$$

The proof will be given later. With the help of these relations we can prove

TheOREM 11. Let $U=\left(U_{\rho}\right), V=\left(V_{\rho}\right)$ be uniform functions on $\mathcal{S}_{n}$. Then

where, if $\rho=\left\{1^{r_{1}} 2^{r_{2}} \cdots\right\}$,

$$
(U, V)=\sum_{|\rho|=n} K_{\rho}(U, V),
$$

$$
\begin{aligned}
K_{\rho}(U, V)= & \frac{1}{z_{\rho}} \frac{1}{c_{\rho}(q)} \sum U_{\rho}\left(\gamma_{11}, \cdots, \gamma_{1 r_{1}} ; \gamma_{21}, \cdots, \gamma_{2 r_{2}} ; \cdots\right), \\
& \cdot \bar{V}_{\rho}\left(\gamma_{11}, \cdots, \gamma_{1 r_{1}} ; \gamma_{21}, \cdots, \gamma_{2 r_{2}} ; \cdots\right),
\end{aligned}
$$


summed over all rows $\gamma_{11}, \cdots, \gamma_{1 r_{1}}, \gamma_{21}, \cdots, \gamma_{2 r_{2}}, \cdots$ such that $\gamma_{d i} \in M\left(\mho_{d}\right)$ $\left(i=1,2, \cdots, r_{d} ; d=1,2, \cdots\right)$.

Proof. By Lemma 4.14 it is enough to prove this for basic uniform functions $U, V$, of respective types $\rho, \sigma$, say. We have then to show

$$
\begin{array}{ll}
(U, V)=0 & \text { if } \rho \neq \sigma, \\
(U, V)=K_{\rho}(U, V) & \text { if } \rho=\sigma .
\end{array}
$$

If $U_{\rho}, V_{\sigma}$ are the principal $\rho$ - and $\sigma$-parts of $U, V$ respectively, and if $c$ is the class $\left(\cdots f^{\nu(f)} \cdots\right)$, we have by the degeneracy rule (18),

$$
\frac{1}{a(c)} U(c) \bar{V}(c)=\frac{1}{a(c)} \sum_{m, m^{\prime}} Q(m, c) Q\left(m^{\prime}, c\right) U_{\rho}\left(\xi^{\rho} m\right) \bar{V}_{\sigma}\left(\xi^{\sigma} m^{\prime}\right),
$$

summed over modes $m, m^{\prime}$ of substitutions respectively of $X^{\rho}, X^{\sigma}$ in to $c$.

The set $W$ of classes isobaric to $c$ consists of all $c^{\prime}=\left(\cdots f^{\prime \prime}(f) \cdots\right)$ such that

$$
\left|\nu^{\prime}(f)\right|=|\nu(f)|,=n(f) \text {, say } \quad(\text { each } f \in F) .
$$

The result of summing (33) over the classes $c$ of $W$ is

$$
I(W)=\sum_{m, m^{\prime}} A\left(m, m^{\prime}\right) U_{\rho}\left(\xi^{\rho} m\right) \bar{V}_{\sigma}\left(\xi^{\sigma} m^{\prime}\right)
$$

where

$$
A\left(m, m^{\prime}\right)=\sum_{\nu(f)} \prod_{f \in F} \frac{1}{a_{\nu(f)}\left(q^{d(f)}\right)} \frac{1}{z_{\rho(m, f)}} \frac{1}{z_{\sigma\left(m^{\prime}, f\right)}} Q_{\rho(m, f)}^{\nu(f)}\left(q^{d(f)}\right) Q_{\sigma\left(m^{\prime}, f\right)}^{\nu(f)}\left(q^{d(f)}\right),
$$

the summation being over all partition-valued functions $\nu(f)$ such that $|\nu(f)|=n(f)(f \in F)$; we can factorise this and obtain

$$
A\left(m, m^{\prime}\right)=\prod_{f \in F} \frac{1}{z_{\rho(m, f)}} \frac{1}{z_{\sigma\left(m^{\prime}, f\right)}} \sum_{|\lambda|=n(f)} \frac{1}{a_{\lambda}\left(q^{d(f)}\right)} Q_{\rho(m, f)}^{\lambda}\left(q^{d(f)}\right) Q_{\sigma\left(m^{\prime}, f\right)}^{\lambda}\left(q^{d(f)}\right) .
$$

According to Theorem 10, this is zero unless $\rho(m, f)=\sigma\left(m^{\prime}, f\right)$ for each $f \in F$, that is, unless $\rho=\sigma$ and $m=m^{\prime}$ (Lemma 4.8). ( $\left.U, V\right)$ is the sum of the $I(W)$, for the different isobaric sets $W$, so this proves (31). Now suppose that $\rho=\sigma$; we get this time

$$
I(W)=\sum_{m}\left\{\prod_{f} \frac{1}{z_{\rho(m, f)}} \frac{1}{c_{\rho(m, f)}\left(q^{d(f)}\right)}\right\} U_{\rho}\left(\xi^{\rho} m\right) \bar{V}_{\rho}\left(\xi^{\rho} m\right) .
$$

We can verify that $\prod_{f \in F} c_{\rho(m, f)}\left(q^{d(f)}\right)=c_{\rho}(q)$, and so

$$
I(W)=\frac{1}{c_{\rho}(q)} \sum_{m}\left\{\prod_{f} \frac{1}{z_{\rho(m, f)}}\right\} U_{\rho}\left(\xi^{\rho} m\right) \bar{V}_{\rho}\left(\xi^{\rho} m\right),
$$


the summation being over all modes $m$ of substitution of $X^{\rho}$ into a class $c$ of $W$. Adding for the different $W$ we get

$$
(U, V)=\frac{1}{c_{\rho}(q)} \sum_{m}\left\{\prod_{f} \frac{1}{z_{\rho(m, f)}}\right\} U_{\rho}\left(\xi^{\rho} m\right) \bar{V}_{\rho}\left(\xi^{\rho} m\right),
$$

summed over all modes $m$ of substitution of $X^{\rho}$. An elementary calculation shows that this is the same as $K_{\rho}(U, V)$ described in the theorem.

Before we prove Theorem 10, we shall construct certain characters of $\mathbb{S}_{n}$, which are basic uniform functions of a given type $\rho=\left\{1^{r_{1} 2^{r_{2}}} \ldots\right\}$. As we remarked at the end of $\S 5$, the character $I_{d}^{k}[1]$, which we now write $J_{d}(k)$, is a basic uniform function on $\mathfrak{G}_{d}$ of type $\{d\}$, with $\{d\}$-part $T_{d, 1}\left(k: \xi_{d 1}\right)$ $=\theta^{k}\left(\xi_{d 1}\right)+\theta^{q k}\left(\xi_{d 1}\right)+\cdots+\theta^{a^{d-1} k}\left(\xi_{d 1}\right)$.

Let $h$ stand for the row $\left(h_{11}, \cdots, h_{1 r_{1}} ; h_{21}, \cdots, h_{2 r_{2}} ; \cdots\right)$ of integers $h_{d i}$, one integer for each part of $\rho$.

Definition 6.2. Let $B=B^{\rho}(h)$ be the character

$$
B^{\rho}(h)=J_{1}\left(h_{11}\right) \circ \cdots \circ J_{1}\left(h_{1 r_{1}}\right) \circ J_{2}\left(h_{21}\right) \circ \cdots \circ J_{2}\left(h_{2 r_{2}}\right) \circ \cdots
$$

of $B_{n}$. By Theorem 7 , this is a basic uniform function of type $\rho$, with $\rho$-part

$$
B_{\rho}=B_{\rho}\left(h: \xi^{\rho}\right)=\prod_{d}\left\{\sum_{1^{\prime} 2^{\prime} \cdots r_{d}^{\prime}} S_{d}\left(h_{d 1}: \xi_{d 1^{\prime}}\right) S_{d}\left(h_{d 2}: \xi_{d 2^{\prime}}\right) \cdots S_{d}\left(h_{d r_{d}}: \xi_{d r_{d}^{\prime}}\right\}\right.
$$

where the summation is over all permutations $1^{\prime} 2^{\prime} \cdots r_{d}^{\prime}$ of $12 \cdots r_{d}$, and $S_{d}(k: \xi)=T_{d, 1}(k: \xi)=\theta^{k}(\xi)+\theta^{a k}(\xi)+\cdots+\theta^{d^{d-1} k}(\xi)$.

$B^{\rho}(h)$ will be called a basic character of type $\rho$.

Proof of Theorem 10. This proceeds by induction on $n$. The case $n=1$ is trivial. If we know that the relations hold for the $Q_{p}^{\lambda}$ when $|\lambda|,|\rho|<n$, we may carry out the calculation in the proof of Theorem 11, in so far as only these relations are required. Examining (34), we see that $Q_{\rho}^{\lambda}$ with $|\lambda|=n$ can only occur if the class $c$ has all its latent roots equal, so that $c=\left(l^{\lambda}\right)$ for some linear polynomial $l(t)=t-\gamma(\gamma \in M(\mathfrak{F}))$. Write

$$
Y_{\rho \sigma}(q)=\sum_{|\lambda|=n} \frac{1}{a_{\lambda}(q)} Q_{\rho}^{\lambda}(q) Q_{\sigma}^{\lambda}(q) \quad(|\rho|,|\sigma|=n),
$$

and then, by (34), if $\rho \neq \sigma$

$$
(U, V)=\frac{1}{z_{\rho} z_{\sigma}} Y_{\rho \sigma}(q) \sum_{\gamma} U_{\rho}(\gamma, \cdots, \gamma ; \gamma, \cdots) \bar{V}_{\sigma}(\gamma, \cdots, \gamma ; \gamma, \cdots)
$$

summed over $\gamma \in M(\mathfrak{F})$, while if $\rho=\sigma$,

$$
(U, V)=K_{\rho}(U, V)+\left\{\frac{1}{z_{\rho}^{2}} Y_{\rho \rho}(q)-\frac{1}{z_{\rho}} \frac{1}{c_{\rho}(q)}\right\} \sum_{\gamma} U_{\rho}(\gamma, \cdots) \bar{V}_{\rho}(\gamma, \cdots)
$$

Let us now take 


$$
\begin{aligned}
& U=B^{\rho}(h)=J_{1}\left(h_{11}\right) \circ \cdots \circ J_{1}\left(h_{1 r_{1}}\right) \circ J_{2}\left(h_{21}\right) \circ \cdots \circ J_{2}\left(h_{2 r_{2}}\right) \circ \cdots, \\
& V=B^{\sigma}(l)=J_{1}\left(l_{11}\right) \circ \cdots \circ J_{1}\left(l_{1 r_{1}}\right) \circ J_{2}\left(l_{21}\right) \circ \cdots \circ J_{2}\left(l_{2 r_{2}}\right) \circ \cdots .
\end{aligned}
$$

Then $\sum_{\gamma} U_{\rho}(\gamma, \cdots, \gamma ; \gamma, \cdots, \gamma ; \cdots) \bar{V}_{\sigma}(\gamma, \cdots, \gamma ; \gamma, \cdots, \gamma ; \cdots)$ $=z_{\rho} z_{\sigma} \sum_{\gamma} \theta^{a}(\gamma) \bar{\theta}^{b}(\gamma)$, where $a=\sum_{d, i} h_{d i}$, and $b=\sum_{d, i} l_{d i}$. By the character relations for the multiplicative group $M(\mathfrak{F})$,

$$
\sum_{\gamma} \theta^{a}(\gamma) \bar{\theta}^{b}(\gamma)=\delta_{a, b}^{1} \cdot(q-1),
$$

where we write

$$
\delta_{a, b}=\left\{\begin{array}{l}
0, \text { if } a \not \equiv b\left(\bmod q^{d}-1\right), \\
1, \text { if } a \equiv b\left(\bmod q^{d}-1\right)
\end{array} \quad(d=1,2, \cdots) .\right.
$$

We have in general

$$
\sum_{\gamma \in M\left(\mathfrak{\mho}_{d}\right)} \theta^{a}(\gamma) \bar{\theta}^{b}(\gamma)=\delta_{a, b}^{d} \cdot\left(q^{d}-1\right) .
$$

Turning now to the case $\rho=\sigma$, we can easily calculate $K_{\rho}(U, V)$ in our present case. By Definition 6.2,

$$
\begin{aligned}
K_{\rho}\left(B^{\rho}(h), B^{\rho}(l)\right)= & \frac{1}{z_{\rho}} \frac{1}{c_{\rho}(q)} \sum_{\gamma d i} \prod_{d} \sum S_{d}\left(h_{d 1}: \gamma_{d 1^{\prime}}\right) \bar{S}_{d}\left(l_{d 1}: \gamma_{d 1^{\prime \prime}}\right) \cdots \\
& \cdot S_{d}\left(h_{d r_{d}}: \gamma_{d r_{d}^{\prime}}\right) \bar{S}_{d}\left(l_{d r_{d}}: \gamma_{d r_{d}^{\prime \prime}}\right)
\end{aligned}
$$

in which the first summation is over rows $\left(\gamma_{11}, \cdots, \gamma_{1 r_{1}} ; \cdots\right)$ as in Theorem 11 , while the second summation is over all pairs $\left(1^{\prime} \cdots r_{d}^{\prime}\right),\left(1^{\prime \prime} \cdots r_{d}^{\prime \prime}\right)$ of permutations of $12 \cdots r_{d}$. We may write this as

$$
\begin{aligned}
& \prod_{d} \frac{1}{d^{r_{d} r_{d} !}} \cdot \frac{1}{\left(q^{d}-1\right)^{r_{d}}} \cdot r_{d} ! \sum_{\gamma d 1}, \cdots, \gamma d r_{d} \sum_{1^{\prime}} \sum_{r_{d}^{\prime}} S_{d}\left(h_{d 1}: \gamma_{d 1}\right) \\
& \cdot \bar{S}_{d}\left(l_{d 1^{\prime}}: \gamma_{d 1}\right) \cdots S_{d}\left(h_{d r_{d}}: \gamma_{d r_{d}}\right) \bar{S}_{d}\left(l_{d r_{d}^{\prime}}: \gamma_{d r_{d}}\right),
\end{aligned}
$$

where the first summation is over all rows $\gamma_{d 1}, \cdots, \gamma_{d r_{d}}$ of elements of $M\left(\mathfrak{F}_{d}\right)$. We see that the term

$$
\sum_{\gamma_{d l^{\prime}}, \cdots, \gamma_{d r_{d}}} S_{d}\left(h_{d 1}: \gamma_{d 1}\right) \bar{S}_{d}\left(l_{d 1^{\prime}}: \gamma_{d 1}\right) \cdots S_{d}\left(h_{d r_{d}}: \gamma_{d r_{d}}\right) \bar{S}_{d}\left(l_{d r_{d}^{\prime}}: \gamma_{d r_{d}}\right)
$$

factorises to give

$$
\prod_{i=1}^{r_{d}} \sum_{\gamma \in M\left(\mathfrak{F}_{d}\right)} S_{d}\left(h_{d i}: \gamma\right) \bar{S}_{d}\left(l_{d i^{\prime}}: \gamma\right)
$$

By (37), 


$$
\sum_{\gamma \in M\left(\mathfrak{F}_{d}\right)} S_{d}(a: \gamma) \bar{S}_{d}(b: \gamma)=d \Delta^{d}(a, b) \cdot\left(q^{d}-1\right),
$$

where

$$
\Delta^{d}(a, b)=\delta_{a, b}^{d}+\delta_{a, b q}^{d}+\cdots+\delta_{a, b q^{d-1}}^{d}
$$

and so we get

$$
K_{p}(U, V)=\prod_{d}\left\{\prod_{1^{\prime} \cdots r_{d}^{\prime}} \Delta^{d}\left(h_{d 1}, l_{d 1^{\prime}}\right) \Delta^{d}\left(h_{d 2}, l_{d 2^{\prime}}\right) \cdots \Delta^{d}\left(h_{d r_{d}}, l_{d r_{d}^{\prime}}\right)\right\} .
$$

This shows in particular that $K_{\rho}$ is an integer.

Since $U, V$ are characters, $(U, V)$ must be an integer. Let us specialise $U, V$ still further, making all $h_{d i}=l_{d i}=0$. It follows then from (35), (36) and what we have just proved, that

$$
Y_{\rho \sigma}(q) \cdot(q-1) \text { and }\left\{Y_{\rho \rho}(q)-\frac{z_{\rho}}{c_{\rho}(q)}\right\} \cdot(q-1)
$$

are integers, and this must be true for each prime-power $q$. Now by Lemmas 2.4 and 4.3 , these are each rational functions in $q$ whose numerator is of smaller degree than the denominator, provided that $n>1$. Thus they tend to zero as $q \rightarrow \infty$. This means that for all sufficiently large prime-power values of $q$ they are zero, and therefore they are identically zero.

Incidentally we have proved

LemMa 6.3. Let $\rho=\left\{1^{r_{1}} 2^{r_{2}} \cdots\right\}, \sigma=\left\{1^{s_{1}} 2^{s_{2}} \ldots\right\}$ be partitions of $n$, and let $h, l$ stand for the rows

$$
\begin{aligned}
& \left(h_{11}, \cdots, h_{1 r_{1}} ; h_{21}, \cdots, h_{2 r_{2}} ; \cdots\right), \text { and } \\
& \left(l_{11}, \cdots, l_{1 r_{1}} ; l_{21}, \cdots, l_{2 r_{2}} ; \cdots\right) \text { respectively. }
\end{aligned}
$$

Let $B^{\rho}(h), B^{\rho}(l)$ be the characters defined by 6.2. Then $\left(B^{\rho}(h), B^{\sigma}(l)\right)=0$ if $\rho \neq \sigma$, and

$$
\left(B^{\rho}(h), B^{\rho}(l)\right)=\prod_{d}\left\{\sum_{1^{\prime}, \ldots r_{d^{\prime}}} \Delta^{d}\left(h_{d 1}, l_{d 1^{\prime}}\right) \cdots \Delta^{d}\left(h_{d r_{d^{\prime}}}, l_{d r_{d^{\prime}}}\right)\right\}
$$

where

$$
\Delta^{d}(a, b)=\delta_{a, b}^{d}+\delta_{a, b q}^{d}+\cdots+\delta_{a, b q^{d-1}}^{d} \quad(d=1,2, \cdots) .
$$

7. The primary irreducible characters. Let $s$ be an integer, and $\pi=\left\{1^{p_{1}} 2^{p_{2}} \cdots\right\}$ a partition. Write $s \cdot \pi$ for the partition $\left\{s^{p_{1}}(2 s)^{p_{2}} \cdots\right\}$.

The basic character $B^{\rho}(h)$ has $\rho$-part $B_{\rho}\left(h: \xi^{\rho}\right)$ given in 6.2. By Theorem 9 the character $I_{s}^{k}[v]$ has $\rho$-part zero unless $\rho=s \cdot \pi$ for some $\pi$, while its $s \cdot \pi$ part is $U_{s \cdot \pi}(k: \xi)=\prod_{e} \prod_{i-1}^{p_{e}} T_{s, e}\left(k: \xi_{s, i}\right)$. 
Lemma 7.1.

$$
B_{8 \cdot \pi}\left(k \frac{\pi}{s}: \xi^{8 \cdot \pi}\right)=z_{\pi} U_{\cdot \cdot \pi}\left(k: \xi^{s \cdot \pi}\right),
$$

where $k \pi / s$ stands for the row $\left(h_{s 1}, \cdots, h_{s p_{1}} ; h_{2 s, 1}, \cdots, h_{2 s, p_{2}} ; \cdots\right)$ in which

$$
h_{s e, i}=k\left(1+q^{s}+\cdots+q^{(e-1) s}\right), \quad\left(i=1,2, \cdots, p_{s} ; e=1,2, \cdots\right) .
$$

This follows from the definitions, using the easily verified identity

$$
S_{s e}\left(k\left(1+q^{s}+\cdots+q^{(e-1) s}: \xi\right)=e T_{s, e}(k: \xi) .\right.
$$

Two uniform functions with the same principal parts must be equal. Thus we have

Lемма 7.2. If $k \pi / s$ is the row defined above, then

$$
I_{s}^{k}[v]=\sum_{|\pi|=v} \frac{1}{z_{\pi}} B^{s \cdot \pi}\left(k \frac{\pi}{s}\right) .
$$

By Definition 6.2,

$$
B^{s \cdot \pi}\left(k \frac{\pi}{s}\right)=Z_{1} \circ \cdots \circ Z_{1} \circ Z_{2} \circ \cdots \circ Z_{2} \circ \cdots
$$

there being $p_{e}$ factors $Z_{s}=J_{s e}\left(k\left(1+q^{s}+\cdots+q^{(e-1) s}\right)(e=1,2, \cdots)\right.$. We may compare (38) with the formula

$$
\{v\}=\sum_{|\pi|=v} \frac{1}{z_{\pi}} P_{\pi}
$$

in which $\{v\}$ represents the Schur function in $n \geqq v$ variables $t_{1}, t_{2}, \cdots, t_{n}$ (this particular Schur function is often written $h_{v}$ and called the homogeneous product sum of degree $v$. See, for example, D. E. Littlewood [9]), $P_{e}=t_{1}^{e}+t_{2}^{e}$ $+\cdots+t_{n}^{e}(e=1,2, \cdots)$, and $P_{\pi}=P_{1}^{p_{1}} P_{2}^{p_{2}} \cdots$ for any partition $\pi=\left\{1^{p_{1}} 2^{p_{2}} \cdots\right\} . P_{1}, P_{2}, \cdots, P_{n}$ are algebraically independent generators of the algebra $\delta$ of symmetric polynomials in $t_{1}, t_{2}, \cdots, t_{n}$ with complex coefficients, and on the other hand the o-product is commutative and associative, so that the mapping

$$
P_{\bullet} \rightarrow Z_{e} \quad(e=1,2, \cdots, n)
$$

defines a homomorphism of $S$ into the algebra $A$ defined in the introduction. To the Schur function $\{v\}=h_{v}$, there corresponds, under this homomorphism, the function $I_{s}^{k}[v]$, by Lemma 7.2.

For a partition $\lambda$ of $v$, the Schur function $\{\lambda\}$ is defined (Littlewood, loc. cit.) by 


$$
\{\lambda\}=\sum_{|\pi|=v} \frac{1}{z_{\pi}} \chi_{\pi}^{\lambda} P_{\pi},
$$

$\chi_{\pi}^{\lambda}$ being characters of the symmetric group $\mathfrak{S}_{v}$, and to this corresponds the class-function on $\mathbb{G}_{v}$

DeFINITION 7.3

$$
I_{*}^{k}[\lambda]=\sum_{|\pi|=0} \frac{1}{z_{x}} \chi_{\pi}^{\lambda} B^{s \cdot \pi}\left(k \frac{\pi}{s}\right) .
$$

We have yet to show that this is a character; the coefficients $\chi_{\pi}^{\lambda} / z_{\pi}$ are not all integers. But we do know (Littlewood, loc. cit.) that $\{\lambda\}$ can be expressed as a rational integral combination of products of the form

$$
h_{v_{1}} h_{v_{2}} \cdots h_{v_{r}} \text {, }
$$

and using the homomorphism of $S$ into $\mathcal{A}$, we have that $I_{s}^{\mathrm{k}}[\lambda]$ is a rational integral combination of characters

$$
I_{*}^{k}\left[v_{1}\right] \circ I_{s}^{k}\left[v_{2}\right] \circ \cdots \circ I_{s}^{k}\left[v_{r}\right]
$$

and is therefore itself a character.

By the degree of a character $\chi$ of $B$ (even if $\chi$ is not a proper character) we mean $\chi(1)$, where 1 is the identity element of $\$\left(S\right.$. The degree of $J_{d}(k)=I_{d}^{k}[1]$ is

$$
J_{d}(k)(1)=\phi_{d-1}(q) \quad \text { (Example (ii), §5). }
$$

By Lemma 2.7 the degree of the basic character $B^{p}(h)$ is

$$
\begin{aligned}
\beta_{\rho} & =\frac{\psi_{n}(q)}{\psi_{1}(q)^{r_{1}} \psi_{2}(q)^{r_{2}} \ldots} \cdot \phi_{0}(q)^{r_{1}} \phi_{1}(q)^{r_{2}} \ldots \\
& =\phi_{n}(q) \cdot e_{\rho}(q),
\end{aligned}
$$

writing

$$
e_{\rho}(q)=\frac{1}{(1-q)^{r_{1}}} \cdot \frac{1}{\left(1-q^{2}\right)^{r_{2}}} \cdots \cdots
$$

Consider now the homomorphism of the algebra $S$ into the field of rational functions in a variable $q$, which is defined by

$$
P_{e} \rightarrow \frac{1}{1-q^{e}} \quad(e=1,2, \cdots, n) .
$$

Under this mapping a certain rational function

$$
\{\lambda: q\}=\sum_{\rho} \frac{1}{z_{p}} \chi_{\rho}^{\lambda} e_{\rho}(q)
$$


corresponds to the Schur function $\{\lambda\}$. Comparing $P_{e}=t_{1}^{e}+t_{2}^{e}+\cdots+t_{n}^{e}$ with the formal expansion

$$
1 /\left(1-q^{e}\right)=1^{e}+q^{e}+\left(q^{2}\right)^{e}+\cdots,
$$

we see that (provided $|\lambda| \leqq n)\{\lambda: q\}$ is just the formal Schur function in the infinity of variables

$$
1, q, q^{2}, \cdots .
$$

D. E. Littlewood (loc. cit., Chapter VII) has calculated these functions explicitly and found that, if $\lambda=\left\{l_{1}, l_{2}, \cdots, l_{p}\right\}$ where $l_{1} \geqq l_{2} \geqq \cdots \geqq l_{p}>0$, then

$$
\{\lambda: q\}=q^{l_{2}+2 l_{3}+\cdots} \prod_{1 \leqq r<s \leqq p}\left(1-q^{l_{r}-l_{s}-r+s}\right) / \prod_{r=1}^{p} \phi_{l r+p-r}(q) .
$$

We may remark that if $\rho=s \cdot \pi$, then $e_{\rho}(q)=e_{\pi}\left(q^{s}\right)$; and then using 7.3, (39), and (40) we have

Lemma 7.4. Let $s, v$ be positive integers, $s v=n$, and $\lambda$ a partition of $v$. Then the degree of the character $I_{s}^{k}[\lambda]$ of $B_{n}$ is

$$
\phi_{n}(q) \cdot\left\{\lambda: q^{s}\right\}
$$

where $\{\lambda: q\}$ is the function defined in (41).

Two special cases are of interest: if $\lambda=\{v\},\{\lambda ; q\}=1 / \phi_{v}(q)$, so that

The degree of $I_{s}^{k}[v]$ is $\phi_{s v}(q) / \phi_{v}\left(q^{s}\right)$;

and if $\lambda=\left\{1_{v}\right\},\{\lambda: q\}=q^{C_{v}, 2 / \phi_{v}}(q)$, so that

The degree of $I_{s}^{k}\left[1^{v}\right]$ is $q^{C_{v}, 2 \phi_{s v}}(q) / \phi_{v}\left(q^{s}\right)$.

The character $I_{1}^{k}\left[1^{n}\right]$, whose degree is $q^{C_{n, 2}}$, has been calculated by Steinberg (13), who was the first to discover characters which are effectively the $I_{1}^{\boldsymbol{k}}[\lambda]$. By examining (41) we have

Lemma 7.5. The degree of $I_{s}^{k}[\lambda]$ has the sign of $(-1)^{(s-1) v}=(-1)^{n-v}$.

Lemma 7.6. Let $s, v$ be positive integers, and $\lambda$ any partition of $v$. Then $\left\|I_{s}^{k}[\lambda]\right\|=1$, if

$$
k, k q, k q^{2}, \cdots, k q^{s-1}
$$

are $s$ distinct residues $\bmod \left(q^{s}-1\right)$.

It is easy to give a direct proof of this, but it is a special case of a Lemma (8.3) which will be proved in the next section. The condition on $k$ which is described is equivalent to the statement $\Delta^{s}(k, k)=1$.

Let $\epsilon$ be a generator of $M\left(\mathfrak{F}^{*}\right)$, and let $\epsilon_{s}=\epsilon^{\left(q^{n t}-1\right) /\left(q^{*}-1\right)}(1 \leqq s \leqq n) . \epsilon_{s}$ is a generator of $M\left(\mathfrak{F}_{s}\right)$. Each nonzero element of $\mathfrak{F}_{s}$ has an expression $\epsilon_{s}^{\boldsymbol{k}}$, and in this, $k$ is uniquely determined $\bmod \left(q^{s}-1\right)$. The condition for $\epsilon_{s}^{k}$ to have the 
degree $s$ is that all its $s$ conjugates $\epsilon_{s}^{\boldsymbol{k}}, \epsilon_{s}^{\boldsymbol{k}}, \cdots, \boldsymbol{\epsilon}_{s}^{\boldsymbol{k}^{a-1}}$ should be distinct, which means that

$$
k, k q, \cdots, k q^{8-1}
$$

are distinct residues $\bmod \left(q^{s}-1\right)$. In this circumstance we shall say that each of the integers (42) is an s-primitive, and that the set (42) is an s-simplex $g$ (or a simplex $g$ of degree $s$ ) with $k, k q, \cdots, k q^{s-1}$ as its roots. The correspondence between the $s$-simplex $g$ and the irreducible polynomial of degree $s$ which has $\epsilon_{s}^{\boldsymbol{k}}, \epsilon_{s}^{k \boldsymbol{q}}, \cdots, \epsilon_{s}^{\boldsymbol{k}_{s}-1}$ as its roots, proves

LemмA 7.7. There are exactly as many simplexes of degree $s$ as there are irreducible polynomials $f \in F$ of degree $s$.

It is easily seen from the definitions 7.3 and 6.2 that $I_{s}^{k}[\lambda]=I_{s}^{k^{\prime}}[\lambda]$ if $k, k^{\prime}$ are roots of the same $s$-simplex $g\left(\right.$ in other words, if $k^{\prime} \equiv k q^{u}\left(\bmod \left(q^{s}-1\right)\right)$ for some integer $u$ ). Thus there is no ambiguity in writing

$$
\left(g^{\lambda}\right)=(-1)^{(8-1) v} I_{s}^{k}[\lambda] .
$$

The lemmas in this section allow us to deduce

Theorem 12. Let $s, v$ be positive integers. Then for each s-simplex $g$, and each partition $\lambda$ of $v$, we have an irreducible character $\left(g^{\lambda}\right)$ of $\mathbb{G}_{s v}$ of degree

$$
(-1)^{(s-1) v} \phi_{s v}(q)\left\{\lambda: q^{s}\right\} \text {. }
$$

Further, $U=\left(g^{\lambda}\right)$ is a uniform function, and if $k$ is any root of $g$, the principal parts are $U_{\rho}=0$, unless all the parts of $\rho$ are divisible by $s$, while if $\rho=s \cdot \pi$, with $\pi=\left\{1^{p_{1}} 2^{p_{2}} \ldots\right\}$,

$$
U_{\rho}\left(\xi^{\rho}\right)=(-1)^{(s-1) v} \chi_{\pi}^{\lambda} \prod_{e} \prod_{i=1}^{p_{e}} T_{s, e}\left(k: \xi_{s e, i}\right) .
$$

The $\left(g^{\lambda}\right)$ may be called the primary irreducible characters.

8. The irreducible characters of $\mathrm{BS}_{n}$. Let $G$ be the set of all $s$-simplexes, for $1 \leqq s \leqq n$, and let $d(g)$ denote the degree of $g \in G$. Our object is the

THEOREM 13. Let $\nu(g)$ be a partition-valued function on $G$ such that

$$
\sum_{g \in G}|\nu(g)| d(g)=n
$$

Then

$$
\left(\cdots g^{\nu(o)} \cdots\right)=\prod_{g \in G}\left(g^{\nu(g)}\right)
$$

( I stands for o-product, and a factor for which $\nu(g)=0$ is omitted) is an irreducible character of $\mathfrak{S}_{n}$, and the only irreducible characters of $\mathscr{S}_{n}$ are those obtained in this way. 
Lemmas 1.1 and 7.7 show that there are exactly as many characters $\left(\cdots g^{\nu(\theta)} \cdots\right)$ as there are classes of $B_{n}$. Therefore we have only to show that these characters are all irreducible and distinct, and then we know there can be no further irreducible characters.

Let us call $\left(\cdots g^{\nu(o)} \cdots\right)$ the "symbol" of a "dual class" $e$. If $g$ is an $s$-simplex with root $k$, and if $\lambda$ is a partition of $v$, it will be convenient to write $I_{s}^{\mathbf{k}}[\lambda]=I_{\theta}[\lambda]$; so that $\left(g^{\lambda}\right)=(-1)^{(\bullet-1) v} I_{o}[\lambda]$. Now define, for a dual class $e=\left(\cdots g^{\nu(g)} \cdots\right)$, the character

$$
I_{\bullet}=\prod_{o \in G} I_{0}[\nu(g)]
$$

This character $I_{0}$ is of course connected with the character $\left(\cdots g^{\nu(\theta)} \cdots\right)$ defined in Theorem 13 by the trivial relation

$$
\begin{aligned}
I_{\theta} & =(-1)^{\Sigma(d(\theta)-1\}|\nu(o)|}\left(\cdots g^{\nu(\theta)} \cdots\right) \\
& =(-1)^{n-\Sigma|\nu(o)|}\left(\cdots g^{\nu(\theta)} \cdots\right)
\end{aligned}
$$

where $\sum$ stands, in each case, for $\sum_{o \in G}$. However $I_{\theta}$ is the more convenient or computation.

For each partition $\rho=\left\{1^{r_{1}} 2^{r_{2}} \ldots\right\}$ of $n$, define the set $Y^{\rho}$ of "dual $\rho$-variables" $y_{d i}^{\rho}=y_{d i}\left(i=1,2, \cdots, r_{d} ; d=1,2, \cdots\right)$, and say that $y_{d i}$ has degree $d\left(y_{d i}\right)=d$. A substitution $\alpha$ of $Y^{\rho}$ is a mapping of $Y^{\rho}$ into $G$ which satisfies

$$
d(y \alpha) \text { divides } d(y) \text {, for each } y \in Y^{\rho} \text {. }
$$

Equivalence, modes, and the partition $\rho(\alpha, g)$ are defined just as in $\S 4$; we have lemmas analogous to 4.7 and 4.8. $\alpha$ is a substitution into the dual class $e=\left(\cdots g^{v_{0}(o)} \cdots\right)$ if and only if

$$
|\rho(\alpha, g)|=\left|\nu_{\odot}(g)\right|
$$

Isobaric dual classes can be defined, as in $\$ 4$.

We depart from the strict analogy with $\$ 4$ when dealing with the "dual $\rho$-roots." We define a set $H^{\rho}$ of $n$ symbols, of which

$$
h_{d i}^{\rho}=h_{d i}, h_{d i} q, \cdots, h_{d i} q^{d-1}
$$

are the "roots" of $y_{d i}^{\rho}=y_{d i}\left(i=1,2, \cdots, r_{d} ; d=1,2, \cdots\right)$. We may think of these as "exponents" of corresponding $\rho$-roots, say $\epsilon_{d}^{h_{d i}}=\xi_{d i}\left(\epsilon_{d}\right.$ is the generator of $M\left(\mathfrak{F}_{d}\right)$ which was defined in $\left.\S 7\right)$. If a substitution $\alpha$ of $X^{\rho}$ takes $\xi_{d i}$ to an element $\gamma=\epsilon_{s}^{c}$ of $M\left(\mathfrak{F}^{*}\right)$ which has degree $s$ ( $s$ divides $\left.d\right)$, the effect in terms of exponents is to take

$$
\xi_{d i}=\stackrel{h d i}{\epsilon} \underset{d}{\text { to }} \gamma=\underset{\epsilon}{\epsilon}=\epsilon_{d}^{c\left(q^{d}-1\right) /\left(q^{d}-1\right)} .
$$

Here $c$ is an $s$-primitive, since $\epsilon_{s}^{c}$ has degree $s$.

We do not propose to use any such explicit correspondence between $H^{p}$ and $\Xi^{\rho}$ as that just described, but give this as explanation of the following 
Definition 8.1. If $\alpha$ is a substitution of $Y^{\rho}$, we define $\alpha$ as a mapping of $H^{\rho}$ into the rational integers, by

$$
h_{d i} \alpha=c_{d i}\left(q^{d}-1\right) /\left(q^{s d i}-1\right),
$$

where $c_{d i}$ is a root of the simplex $y_{d i} \alpha$, and $s_{d i}$ is the degree of $y_{d i} \alpha$.

Conversely any such mapping of $H^{p}$, in which for each $i=1,2, \cdots, r_{d}$, $d=1,2, \cdots, c_{d i}$ is an $s_{d i}$-primitive and $s_{d i}$ divides $d$, defines a substitution of $Y^{p}$.

Finally a dual $\rho$-function is a function $U_{\rho}\left(y_{11}, \cdots, y_{1 r_{1}} ; \cdots\right)$ $=U_{\rho}\left(h_{11}, \cdots, h_{1 r_{1}} ; \cdots\right)$ which takes a value $U_{\rho}\left(y^{\rho} \alpha\right)=U_{\rho}\left(h^{\rho} \alpha\right)=U_{\rho}\left(h^{\rho} m\right)$ $=U_{\rho}\left(h_{11} \alpha, \cdots, h_{1 r_{1}} \alpha ; \cdots\right)$ for each substitution $\alpha$ of $Y^{\rho}$, this value depending only on the mode $m$ of $\alpha$. An example of a dual $\rho$-function is $B_{\rho}\left(h: \xi^{\rho}\right)$ defined in 6.2 (regarded as function of the $h_{d i}$ ).

Lemma 8.2. Let $e=\left(\cdots g^{(\theta)} \cdots\right)$ be a dual class of $\mathcal{B S}_{n}$, then

$$
I_{e}=\sum_{\rho} \sum_{m} \chi(m, e) B^{\rho}\left(h^{\rho} m\right)
$$

summed over all partitions $\rho$ of $n$, and all modes $m$ of substitution of $Y^{\rho}$ into $e$, and where

$$
\chi(m, e)=\prod_{\sigma \in G} \frac{1}{z_{\rho(m, \theta)}} \chi_{\rho(m, \theta)}^{p(o)} .
$$

This is a dual "degeneracy rule," which enables us to compute the character $I_{0}$, and thence $\left(\cdots g^{\nu(o)} \cdots\right)$, in terms of the basic characters $B^{\rho}\left(h^{\rho}\right)$.

Proof. Write $k(g)$ for a root of $g \in G$, and $d(g)$ for the degree of $g$. Definition 7.3 gives

$$
I_{o}[\nu(g)]=I_{k(o)}^{d(o)}[\nu(g)]=\sum_{|\pi|=|\nu(g)|} \frac{1}{z_{\pi}} \chi_{\pi}^{\gamma(\theta)} B^{d(g) \cdot \pi}\left(k(g) \frac{\pi}{d(g)}\right) .
$$

Substituting this in (43) we have

$$
I_{0}=\sum_{\pi(g)} \prod_{\theta \in g} \frac{1}{z_{\pi(g)}} \chi_{\pi(\theta)}^{(\rho)} B^{d(\theta) \cdot \pi(\theta)}\left(k(g) \frac{\pi(g)}{d(g)}\right),
$$

where $\Pi$ is a o-product, and the summation is over all partition-valued functions $\pi(g)$ on $G$, which are such that $|\pi(g)|=|\nu(g)|(g \in G)$. By (T2) and the analogue of Lemma 4.8 , such functions $\pi(g)$ are exactly the functions $\rho(m, g)$ taken over all partitions $\rho$ of $n$, and all modes $m$ of substitution of $Y^{\rho}$ into $e$. Finally, we can see that if $\pi(g)=\rho(m, g)$, then

$$
\prod_{\rho \in G} B^{d(\boldsymbol{\theta}) \cdot \pi(\rho)}\left(k(g) \frac{\pi(g)}{d(g)}\right)=B^{\rho}\left(h^{\rho} m\right),
$$

by writing each character $B^{d(\theta) . \pi(\theta)}$ on the left as a product of $J$ 's, according 
to Definition 6.2. (We see here the connection between Definition 8.1, and the symbol $k \pi / s$ defined in Lemma 7.1.) This proves the lemma.

Lemma 8.3. Let $e=\left(\cdots g^{\nu(\theta)} \cdots\right), e^{\prime}=\left(\cdots g^{\nu(g)} \cdots\right)$ be two dual classes of $\mathbb{S}_{n}$. Then

$$
\left(I_{e}, I_{e^{\prime}}\right)=0 \text { if } e \neq e^{\prime},
$$

while $\left(I_{e}, I_{e}\right)=\left\|I_{e}\right\|=1$.

Proof. From Lemma 8.2,

$$
\left(I_{e}, I_{e^{\prime}}\right)=\sum_{\rho, m} \sum_{\rho^{\prime}, m^{\prime}} \chi(m, e) \chi\left(m^{\prime}, e^{\prime}\right)\left(B^{\rho}\left(h^{\rho} m\right), B^{\rho^{\prime}}\left(h^{\rho^{\prime}} m^{\prime}\right)\right),
$$

summed over all pairs $\rho, \rho^{\prime}$ of partitions of $n$, and all pairs $m, m^{\prime}$ of modes of substitutions of $Y^{p}, Y^{\rho^{\prime}}$ respectively in to $e, e^{\prime}$. By Lemma 6.3,

$$
\left(B^{\rho}\left(h^{\rho} m\right), B^{\rho^{\prime}}\left(h^{\rho^{\prime}} m^{\prime}\right)\right)=0, \quad \text { unless } \rho=\rho^{\prime} .
$$

If $\rho=\rho^{\prime}$, and if $\alpha, \alpha^{\prime}$ are substitutions of the modes $m, m^{\prime}$ respectively, then

$\left(B^{\rho}\left(h^{\rho} m\right), B^{\rho}\left(h^{\rho} m^{\prime}\right)\right)$

$$
=\prod_{d}\left\{\sum_{1^{\prime}, \cdots r_{d}^{\prime}} \Delta^{d}\left(h_{d 1} \alpha, h_{d 1^{\prime}} \alpha^{\prime}\right) \cdots \Delta^{d}\left(h_{d r_{d}} \alpha, h_{d r_{d}^{\prime}} \alpha^{\prime}\right)\right\} .
$$

If, for given $i, i^{\prime}$, the simplexes $g=y_{d i} \alpha, g^{\prime}=y_{d i}{ }^{\prime} \alpha^{\prime}$ have degrees $s, s^{\prime}$ respectively, then according to our convention (8.1)

$$
h_{d i} \alpha=c\left(q^{d}-1\right) /\left(q^{s}-1\right), \quad h_{d i^{\prime}} \alpha^{\prime}=c^{\prime}\left(q^{d}-1\right) /\left(q^{s \prime}-1\right),
$$

where $c, c^{\prime}$ are roots of $g, g^{\prime}$ respectively.

We remark now that $\Delta^{d}(a, b)$, by its definition (see Lemma 6.3), is zero unless $\epsilon_{d}^{a}$ and $\epsilon_{d}^{b}$ are conjugate. Now $\epsilon_{d}^{h_{d i} \alpha}=\epsilon_{s}^{c}$, and $\epsilon_{d}^{h_{d i^{\prime}} \alpha^{\prime}}=\epsilon_{s^{\prime}}^{\epsilon^{\prime}}$. These cannot be conjugate unless, first, $s=s^{\prime}$ (for $s$ and $s^{\prime}$ are their respective degrees), and further, $c \equiv c^{\prime} q^{u}\left(\bmod q^{s}-1\right)$ for some integer $u$; that is, unless $g=g^{\prime}$. If $g=g^{\prime}$ we find very easily that

$$
\Delta^{d}\left(h_{d i} \alpha, h_{d i^{\prime}} \alpha^{\prime}\right)=d / s=d / d(g) .
$$

Thus (45) is zero unless for each $d=1,2, \cdots$, there is some permutation $1^{\prime} 2^{\prime} \cdots r_{d}^{\prime}$ for which $y_{d i} \alpha=y_{d i^{\prime}} \alpha^{\prime}\left(i=1,2, \cdots, r_{d}\right)$. This is exactly the condition for $\alpha, \alpha^{\prime}$ to be equivalent. Therefore (45) is zero unless $m=m^{\prime}$, and this means that $e, e^{\prime}$ must be isobaric $\left(|\nu(g)|=\left|\nu^{\prime}(g)\right|\right.$ for each $\left.g \in G\right)$, because there exists a substitution which is into both.

When $m=m^{\prime}$, we find by some elementary calculations from (45) and (46), that

$$
\left(B^{\rho}\left(h^{\rho} m\right), B^{\rho}\left(h^{\rho} m\right)\right)=\prod_{\rho \in G} z_{\rho(m, o)} .
$$

We have therefore 


$$
\begin{aligned}
\left(I_{e}, I_{e^{\prime}}\right) & =\sum_{\rho, m} \chi(m, e) \chi\left(m, e^{\prime}\right) \prod_{\rho \in G} z_{\rho(m, g)} \\
& =\sum_{\rho, m} \prod_{g} \frac{1}{z_{\rho(m, g)}} \chi_{\rho(m, g)}^{\nu(g)} \chi_{\rho(m, g)}^{\nu^{\prime}(g)} .
\end{aligned}
$$

By the analogue of Lemma 4.8, every partition-valued function $\pi(g)$ on $G$ which is such that $|\pi(g)|=|\nu(g)|\left(=\left|\nu^{\prime}(g)\right|\right)$ for each $g \in G$, occurs as $\rho(m, g)$ for some $\rho, m$. Therefore we may factorize the above expression, and obtain

$$
\left(I_{e}, I_{e^{\prime}}\right)=\prod_{o \in G} \sum_{|\pi|=|\nu(o)|} \frac{1}{z_{\pi}} \chi_{\pi}^{\nu(o)} \chi_{\pi}^{\nu^{\prime}(g)},
$$

and the character relations for the $\chi_{\pi}^{\nu}$ then show that this is zero unless $\nu(g)=\nu^{\prime}(g)$ for each $g \in G$, that is, unless $e=e^{\prime}$; while $\left(I_{e}, I_{e}\right)=1$.

This lemma, taken together with (44), proves Theorem 13, and this completes our investigation. We collect the main results in a final

THEOREM 14. For each partition $\rho=\left\{1^{r_{1}} 2^{r_{2}} \ldots\right\}$ of $n$, write

$$
\begin{aligned}
h^{\rho} & =\left(h_{11}, \cdots, h_{1 r_{1}} ; h_{21}, \cdots, h_{2 r_{2}} ; \cdots\right), \\
\xi^{\rho} & =\left(\xi_{11}, \cdots, \xi_{1 r_{1}} ; \xi_{21}, \cdots, \xi_{2 r_{2}} ; \cdots\right),
\end{aligned}
$$

and let $B_{\rho}\left(h^{\rho}: \xi^{\rho}\right)$ be the function (Definition 6.2)

$$
B_{\rho}\left(h^{\rho}: \xi^{\rho}\right)=\prod_{d}\left\{\sum_{1^{\prime}, \ldots r_{d}^{\prime}} S_{d}\left(h_{d 1}: \xi_{d 1^{\prime}}\right) \cdots S_{d}\left(h_{d r}: \xi_{d r_{d}^{\prime}}\right)\right\},
$$

where

$$
S_{d}(h: \xi)=\theta^{h}(\xi)+\theta^{\alpha h}(\xi)+\theta^{a^{2} h}(\xi)+\cdots+\theta^{a^{d-1} h(\xi),}
$$

and $\theta$ is a generating character of $M\left(\mathfrak{F}^{*}\right)$. For given integers $h_{11}, \cdots, h_{1 r_{1}}$; $h_{21}, \cdots, h_{2 r_{2}}, \cdots$, the basic uniform function (Definitions $\left.4.12,4.13\right) B^{p}(h)$ of type $\rho$ which has $B_{\rho}\left(h: \xi^{\rho}\right)$ as its $\rho$-part, is a character of $\mathbb{B}_{n}$. Each irreducible character of $\mathrm{B}_{n}$ is characterized by a partition-valued function $\nu(g)$ on the set $G$ of simplexes $(\$ 7)$ of degrees $\leqq n$, which satisfies

$$
\sum_{o \in G}|\nu(g)| d(g)=n,
$$

and conversely each such function $\nu(g)$ determines an irreducible character $\left(\cdots g^{\nu(o)} \cdots\right)$ which is given (Lemma 8.2, equation (44)) by

$$
\left(\cdots g^{\nu(o)} \cdots\right)=(-1)^{n-\Sigma|\nu(o)|} \sum_{\rho, m} \chi(m, e) B^{\rho}\left(h^{\rho} m\right) \text {, }
$$

which expresses it as a linear combination of basic characters (the definitions of $\chi(m, e), B^{\rho}\left(h^{\rho} m\right)$ are given in $\left.\S 8\right)$. This character may also be expressed (Theorem 13) as a o-product (see the introduction) of primary irreducible characters (Theorem 12, §7) 


$$
\left(\cdots g^{\nu(o)} \cdots\right)=\prod_{g \in G}\left(g^{\nu(o)}\right),
$$

and its degree (Lemmas $2.7,7.4$ ) is

$$
\psi_{n}(q) \prod_{\rho \in G}(-1)^{|\nu(\theta)|}\left\{\nu(g): q^{d(\theta)}\right\}
$$

where $\psi_{n}(q)=\left(q^{n}-1\right)\left(q^{n-1}-1\right) \cdots(q-1)=(-1)^{n} \phi_{n}(q)$, and, if the parts of $\lambda$, written in descending order, are $l_{1}, l_{2}, \cdots, l_{p}$,

$$
\{\lambda: q\}=q^{l_{2}+2 l_{s}+\cdots} \prod_{1 \leqq r<s \leqq p}\left(1-q^{l_{r}-l_{s}-r+s}\right) / \prod_{r=1}^{p} \phi_{l_{r}+p-r}(q) .
$$

REMARKS. (1) If $m=\sum r_{i}$ is the number of parts of $\rho$, then $(-1)^{n-m} B^{p}(h)$ is itself an irreducible character, provided that, for each $d=1,2, \cdots$, $h_{d 1}, h_{d 2}, \cdots, h_{d_{d}}$ are $d$-primitives, and are the roots of distinct $d$-simplexes $g_{d 1}, g_{d 2}, \cdots, g_{d r_{d}}$, respectively. For, according to $(47),(-1)^{n-m} B^{p}(h)$ is the irreducible character described by the "principal" dual class

$$
\left(g_{11} \cdots g_{1 r_{1}} g_{21} \cdots g_{2 r_{2}} \cdots\right) \text {. }
$$

For general values of the $h_{d i}$, this character is of course not irreducible.

(2) Dual class "types" can be defined in the same way as class types were defined in $\$ 1$. For example, all the "principal characters" (48) (for a given partition $\rho$ ) are of the same type. It is quite easy to set up systems of "type variables," both for nonprincipal types, and for nonprincipal dual types, and to show, using the two degeneracy rules (18) and (47), that all the values of characters of a given type, at classes of a given type, are expressible by a single function in these variables. For example, the value of any character (48) at any principal class of type $\rho$ is obtained by suitably specializing the variables in the formula $(-1)^{m-n} B_{\rho}\left(h^{\rho}: \xi^{\rho}\right)$. All the characters of a given type have the same degree.

(3) Linear characters. A linear character $L=\left(\cdots g^{\nu(\theta)} \cdots\right)$ must be primary, for by Lemma 2.6 no o-product of more than one character can have degree 1 . Let $L=\left(g^{\lambda}\right)$, for an $s$-simplex $g$, and a partition $\lambda$ of $v$, say. The degree $(-1)^{(s-1) v} \phi_{n}(q)\left\{\lambda: q^{s}\right\}$ of $L$ is soon seen to be a polynomial in $q$, multiplied by $q^{s\left(l_{2}+2 l_{3}+\ldots\right)}($ see $[41, \S 7])$, and if this is unity, we must have $l_{2}=l_{3}=\ldots$ $=0$; that is, $\lambda=\{v\}$. By one of the special cases mentioned below Lemma 7.4, the degree of $L$ is now

$$
(-1)^{(-1) v} \phi_{s v}(q) / \phi_{v}\left(q^{s}\right)=\psi_{s v}(q) / \psi_{v}\left(q^{s}\right) .
$$

If $q>2, \psi_{s v}(q) / \psi_{v}\left(q^{\circ}\right)=1$ only if $s=1$. If $q=2$ there is also the case $s=2$, $v=1$. The character $L=I_{1}^{k}[n]$ has been found (see example at the end of $\S 3$ ) to have value $\theta^{k}(\operatorname{det} A)$, at an element $A \in \mathcal{S}_{n}$. We have then the well-known result

If $q>2$, or if $n>2$, the only linear characters of $G L(n, q)$ are $\theta^{k}(\operatorname{det} A)$ $(k=0,1, \cdots, q-2)$. 
The group $G L(2,2)$, which is isomorphic to the symmetric group $\mathfrak{S}_{3}$, has the further linear character $-I_{2}^{1}[1]$.

Appendix. Tables of $Q_{\rho}^{\lambda}(q)$. From the definition (4.2) we may soon verify the following special cases of $Q_{\rho}^{\lambda}(q)(|\lambda|=|\rho|=n)$ :

$$
\begin{array}{rlr}
Q_{\rho}^{\{n\}}=1 & (|\rho|=n), \\
Q_{\rho}^{\left\{1^{n}\right\}}=\beta_{\rho}=\phi_{n}(q) \frac{1}{(1-q)^{r_{1}}} \frac{1}{\left(1-q^{2}\right)^{r_{2}}} \cdots & \left(\rho=\left\{1^{\left.\left.r_{1} 2^{r_{2}} \cdots\right\}\right),}\right.\right. \\
Q_{\{n\}}^{\lambda}=k(\lambda, q) & (|\lambda|=n) .
\end{array}
$$

Lemma 4.4 provides a method for calculating in succession the tables of $Q_{\rho}^{\lambda}$, for $n=1,2,3, \cdots$.

From Theorem 4 of $\mathrm{P}$. Hall, it is easy to show that the symmetric group character $\chi_{\rho}^{\lambda}$ is the coefficient of $q^{n \lambda}$ in $Q_{\rho}^{\lambda}(q)$.

$n=1$.

$$
Q\{1\}=1 \text {. }
$$

\begin{tabular}{|c|c|c|c|c|c|}
\hline 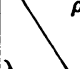 & $\{14\}$ & $\left\{21^{2}\right\}$ & $\{31\}$ & $\{4\}$ & $\left\{2^{2}\right\}$ \\
\hline$\{4\}$ & 1 & 1 & 1 & 1 & 1 \\
\hline$\{31\}$ & $3 q+1$ & $q+1$ & 1 & $1-q$ & $1-q$ \\
\hline$\left\{2^{2}\right\}$ & $(2 q+1)(q+1)$ & $q+1$ & $1-q^{2}$ & $1-q$ & $1-q+2 q^{2}$ \\
\hline$\left\{21^{2}\right\}$ & $\begin{array}{l}\left(3 q^{2}+2 q+1\right) \\
(q+1)\end{array}$ & $-q^{3}+q^{2}+q+1$ & $1-q^{2}$ & $(1-q)\left(1-q^{2}\right)$ & $(1-q)\left(1+q^{2}\right)$ \\
\hline$\left\{1^{4}\right\}$ & $\begin{array}{l}\left(q^{3}+q^{2}+q+1\right) \\
\left(q^{2}+q+1\right)(q+1)\end{array}$ & $\left(q^{2}+q+1\right)\left(1-q^{4}\right)$ & $\left(1-q^{2}\right)\left(1-q^{4}\right)$ & $\begin{array}{l}(1-q)\left(1-q^{2}\right) \\
\left(1-q^{3}\right)\end{array}$ & $\begin{array}{l}(1-q)\left(1+q^{2}\right) \\
\left(1-q^{3}\right)\end{array}$ \\
\hline
\end{tabular}

$n=2$.

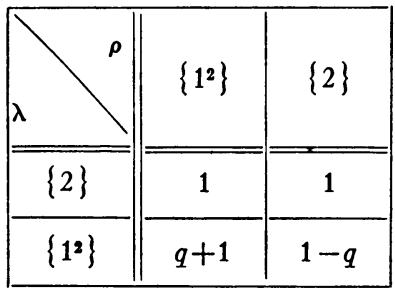

$n=3$.

\begin{tabular}{|c||c|c|c|}
\hline$\lambda$ & $\left\{1^{3}\right\}$ & $\{21\}$ & $\{3\}$ \\
\hline \hline$\{3\}$ & $=1$ & 1 & 1 \\
\hline$\{21\}$ & 1 & $1-q$ \\
\hline$\left\{1^{3}\right\}$ & $\begin{array}{c}\left(q^{2}+q+1\right) \\
(q+1)\end{array}$ & $1-q^{3}$ & $(1-q)\left(1-q^{2}\right)$ \\
\hline
\end{tabular}

$n=4$. 


\begin{tabular}{|c|c|c|c|c|c|c|c|}
\hline$\approx$ & - & I & I & \begin{tabular}{l}
$\approx$ \\
1 \\
\multirow{\sigma}{*}{} \\
1 \\
$=$
\end{tabular} & \begin{tabular}{l} 
ज्ञ \\
1 \\
\multirow{\sigma}{*}{} \\
1 \\
$=$
\end{tabular} & 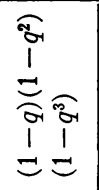 & 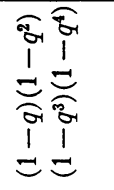 \\
\hline$\widetilde{\widetilde{d}}$ & - & i & $\begin{array}{l}\bar{f} \\
\text { o } \\
\text { of }\end{array}$ & 1 & \begin{tabular}{l} 
o \\
\pm \\
\multirow{્}{*}{} \\
1 \\
$=$
\end{tabular} & $\begin{array}{l}\text { a } \\
1 \\
\vec{\sigma} \\
1 \\
=\end{array}$ & 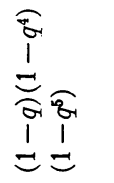 \\
\hline$\tilde{\vec{\Xi}}$ & - & - & $\begin{array}{l}\text { of } \\
\pm\end{array}$ & $\begin{array}{l}\mp \\
\text { a } \\
+ \\
+ \\
\text { J } \\
1\end{array}$ & $\begin{array}{l}\text { b } \\
+ \\
0 \\
0 \\
1 \\
o \\
0 \\
+1\end{array}$ & 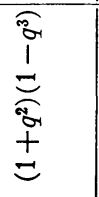 & 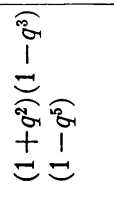 \\
\hline$\tilde{\Xi}$ & - & - & ${ }_{1}^{a-1}$ & I & $\begin{array}{r}\text { or } \\
1 \\
\text { ot } \\
1 \pm \\
= \pm\end{array}$ & 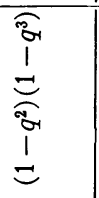 & 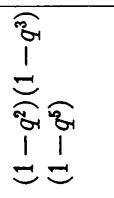 \\
\hline$\stackrel{\widetilde{\omega}}{\sim}$ & - & $\bar{t}_{\text {or }}$ & $\begin{array}{l}\overrightarrow{+} \\
\text { or } \\
+ \\
o \\
1\end{array}$ & $\underset{\sigma}{ \pm}$ & 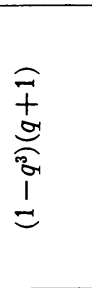 & 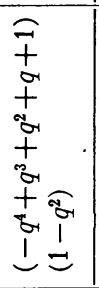 & 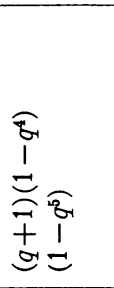 \\
\hline$\stackrel{\widetilde{m}}{\stackrel{\sim}{\Delta}}$ & - & $\underset{d}{+}$ & 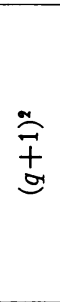 & 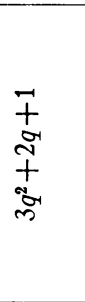 & 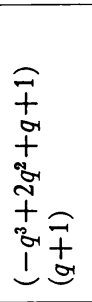 & 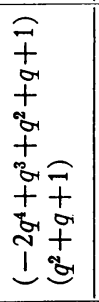 & 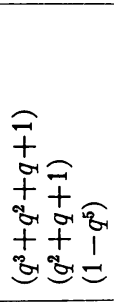 \\
\hline$\stackrel{\Xi}{\varrho}$ & - & $\overrightarrow{+}$ & $\begin{array}{l}\overrightarrow{+} \\
\text { tr } \\
+ \\
\text { or }\end{array}$ & 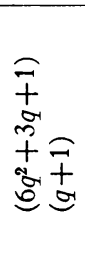 & 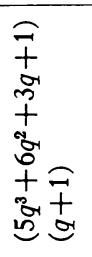 & 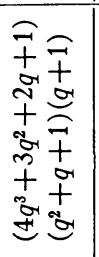 & 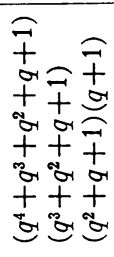 \\
\hline & $\approx$ & $\bar{\Xi}$ & $\widetilde{\sim}$ & $\underset{\sim}{\tilde{N}}$ & $\tilde{\vec{\Xi}}$ & $\stackrel{\widetilde{m}}{\stackrel{\sim}{\sim}}$ & $\stackrel{\tilde{D}}{=}$ \\
\hline
\end{tabular}




\section{BIBLIOGRAPHY}

1. R. Brauer, A characterization of the characters of groups of finite order, Ann. of Math. vol. 57 (1953) pp. 357-377.

2. R. Brauer and C. Nesbitt, On the modular representations of groups of finite order I, University of Toronto Studies, no. 4, 1937.

3. G. Frobenius, Über Relationen zwischen den Charakteren einer Gruppe und denen ihrer Untergruppen, Sitz. Berlin. Akad. (1898) pp. 501-515. pp. $330-339$.

4. - Über die Komposition der Charaktere einer Gruppe, Sitz. Berlin Akad. (1899)

5. - Über die Charaktere der Symmetrischen Gruppe, Sitz. Berlin Akad. (1900) pp. 516-534.

6. P. Hall, A contribution to the theory of groups of prime-power order, Proc. London Math. Soc. vol. 36 (1933) pp. 29-95.

7. - Abelian p-groups and related modules (unpublished).

8. H. Jordan, Group-characters of various types of linear groups, Amer. J. Math. vol. 29 (1907) pp. 387-405.

9. D. E. Littlewood, The theory of group characters and matrix representations of groups, Oxford, 1940.

10. I. Schur, Untersuchungen über die Darstellung der endlichen Gruppen durch gebrochene lineare Substitutionen, J. Reine Angew. Math. vol. 132 (1907) pp. 85-137.

11. A. Speiser, Die Theorie der Gruppen von endlicher Ordnung, 3d ed., New York, 1945.

12. R. Steinberg, The representations of $\mathrm{GL}(3, \mathrm{q}), \mathrm{GL}(4, \mathrm{q}), \operatorname{PGL}(3, \mathrm{q})$ and $\operatorname{PGL}(4, \mathrm{q})$, Canadian Journal of Mathematics vol. 3 (1951) pp. 225-235.

13. - A geometric approach to the representations of the full linear group over a Galois field, Trans. Amer. Math. Soc. vol. 71 (1951) pp. 274-282.

The University, Manchester, England. 